\title{
High Resolution and Fast Scanning SQUID Based Non-destructive Inspection System of Niobium Sheets for SRF Cavities
}

\author{
Closeout Report 2008 \\ Prepared For The US Department of Energy
}

June 8, 2008

Author: Quan-Sheng Shu

\section{GRANT SPONSORED BY THE OFFICE OF SCIENCE Work Performed Under Contract No. DE-FG02-07ER84852}

These SBIR/STTR data are furnished with SBIR/STTR right under Grant DE-FG0207ER84852. For a period of 4 years after acceptance of all items to be delivered under this grant, the Government agrees to use these data for Government purposes only, and they shall not be disclosed outside the Government (including disclosure for procurement purposes) during such period without permission of the grantee, except that, subject to the foregoing use and disclosure prohibitions, such data may be disclosed for use by support contractors. After the aforesaid 4-year period the Government has a royalty-free license to use, and to authorize others to use on its behalf, these data for Government purposes but is relieved of all disclosure prohibitions and assumes no liability for unauthorized use of this data by third parties. This notice shall be affixed to any reproductions of these data in whole or in part.

Prepared by: AMAC International Inc., 12050 Jefferson Ave, Newport News, VA 23606

$\mathrm{Ph}$ : 757-249-3595, Fax: 757-249-3594

DISCLAIMER 
This report was prepared as an account of work sponsored by an agency of the United States Government. Neither the United States Government nor any agency thereof, nor any of their employees, makes any warranty, express or implied, or assumes any legal liability or responsibility for the accuracy, completeness, or usefulness of any information, apparatus, product, or process disclosed, or represents that its use would not infringe privately owned rights. Reference herein to any specific commercial product, process, or service by trade name, trademark, manufacturer, or otherwise does not necessarily constitute or imply its endorsement, recommendation, or favoring by the United States Government or any agency thereof. The views and opinions of authors expressed herein do not necessarily state or reflect those of the United States Government or any agency thereof.

ABSTRACT (Key words: SQUID-based scanning system, nondestructive testing, SRF cavities)

Applications in high energy physics accelerators and other fields require the use of thousands of superconducting RF (SRF) cavities that are made of high purity $\mathrm{Nb}$ material and the purity of niobium is critical for these cavities to reach the highest accelerating fields. Tantalum is the most prolific of metal inclusions, which can cause thermal breakdown and prevent the cavities from reaching their theoretical performance limits of 45-50 MV/m, and DOE Labs are searching for a technology that could detect small impurities in superconducting $\mathrm{Nb}$ sheets reaching the highest possible accelerating fields. The proposed innovative SQUID-based Nondestructive system can scan Niobium sheets used in the manufacturing of SRF cavities with both high speed and high resolution.

A highly sensitive SQUID system with a gradiometer probe, non-magnetic dewar, data acquisition system, and a scanning system will be developed for fast detection of impurities in plannar $\mathrm{Nb}$ sheets. In phase I, we will modify our existing SQUID-based eddy current system to detect 100 micron size Ta defects and a great effort will focus on achieving fast scanning of a large number of niobium sheets in a shorter time and with reasonable resolution. An older system operated by moving the sample $1 \mathrm{~mm}$, stopping and waiting for 1-2 seconds, then activating a measurement by the SQUID after the short settle time is modified. A preliminary designed and implemented a SQUID scanning system that is fast and is capable of scanning a $30 \mathrm{~cm} \times 30 \mathrm{~cm} \mathrm{Nb}$ sheet in 15 minutes by continuously moving the table at speeds up to $10 \mathrm{~mm} / \mathrm{s}$ while activating the SQUID at $1 \mathrm{~mm}$ interval is modified and reached the Phase I goal of $100 \mu \mathrm{m}$ resolution. We have successfully demonstrated the feasibility that a fast speed SQUID scanner without sacrificing the resolution of detection can be done, and a data acquisition and analysis system is also preliminary developed.

The SQUID based scanner will help reach the highest accelerating field in SRF cavities that will provide a considerable cost reduction for new accelerators and for upgrades of existing accelerators. This will be realized either by reducing the length required for the SRF cavities or by the installation of higher gradient cavities for energy upgrades in the same space. The SQUID based scanner will also be used for the detection of defects on the surface, inside the bulk or at the back side of metallic sheets in other industries. 


\section{TABLE OF CONTENTS}

$\begin{array}{ll}\text { C Anticipated Public Benefits } & 10\end{array}$

$\begin{array}{ll}\text { D Phase I Objective } & 11\end{array}$

E Phase I Schedule $\quad 11$

F Related R\&D 12

G Phase I R\&D and Accomplishments 13

H Phase I Conclusion 30

I Reference 31

J Attachment: User’s Instruction 32 


\section{A. IDENTIFICATION AND SIGNIFICANCE}

Applications in high energy physics and free electron lasers require the use of superconducting $\mathrm{RF}$ cavities (SRF) that are made of high purity $\mathrm{Nb}$ material. In the operation of these SRF cavities, the purity of niobium is critical for these cavities to reach the highest gradient fields. Tantalum is the most prolific of metal inclusions and can cause areas of thermal breakdown. Therefore, a method of nondestructive testing is needed to detect small tantalum inclusions in $\mathrm{Nb}$ sheets that are used in making the SRF cavities. The goal of this research work is to improve the techniques of using Superconducting Quantum Interference Devices (SQUIDs) and employing eddy-current techniques to nondestructively detect tantalum inclusions as small as 25 microns in flat sheets of niobium in a fast way. Presently available SQUID Nondestructive Testing (NDT) systems are limited to detection of 100 micron and larger inclusions (for Ta inclusions).

In fabricating SRF cavities, flat sheets are formed into dish-shaped half-cells that are machined and chemically etched before being beam welded to form a complete RF cavity. There is a great need to reliably scan these flat sheet at reasonable and practical speeds and detect small defects $(<50$ micron) before they are used in forming the half cells. Presently there are no Nondestructive Testing (NDT) systems available to inspect flat Niobium sheets that are capable of detecting Tantalum impurities below 100 micrometer in diameter. The availability of this NDT equipment to inspect flat sheet will allow the elimination of defective sheets before the expensive forming and machining of the cavity half-cells. The scanning SQUID system that we propose to develop will provide a fast and reliable NDT testing down to 25 microns $\mathrm{Ta}$ inclusions in $\mathrm{Nb}$ sheets.

SRF cavities used in accelerators have ranged from few cavities to several hundred cavities. The proposed ILC would utilize more than 10,000 SRF cavities in two 10-Km-active-length LINACs. Accelerating gradients of about $35-\mathrm{MV} / \mathrm{m}$ have been reached in several cavities at DESY, the German facility. Efforts are focused on overcoming the most serious obstacles, field emission (FE) and thermal breakdown (TB), which prevent SRF cavities from reaching the theoretical performance limits of $40-50 \mathrm{MV} / \mathrm{m}$ for these $\mathrm{Nb}$ cavities. The $\mathrm{FE}$ and $\mathrm{TB}$ are direct result of the presence of inclusions, such as tantalum. A few number of Ta inclusions per resonator could already lead to a great reduction in the maximum electric field strength realized in these cavities. The fabrication of Superconducting resonators from planar niobium sheets is very costly and there is great need for a measurement procedure that can reliably test the niobium sheets and eliminate defective ones before they are used in the fabrication half cells.

Eddy current techniques that use conventional magnetic sensors to detect defects in metals have the disadvantage of using high frequencies and therefore are useful in only detecting surface defects. Eddy current systems that use SQUID sensors have the advantage of using low frequencies for the excitation currents, which allows the system to detect flaws deep into the material and more importantly, the back side of $\mathrm{Nb}$ sheets. In addition, SQUIDs are the most sensitive detector of magnetic flux with unparallel sensitivity, bandwidth, spatial resolution, 
and fempto-tesla field resolution. Because of the high sensitivity to magnetic fields and the use of low frequency excitation current, SQUID based systems have a tremendous advantage in detecting defects at the surface, inside of, and on the back side the $\mathrm{Nb}$ sheets.

The innovative instrument proposed by AMAC and its collaborators is a SQUID-based eddy current detector for scanning a large number of niobium sheets at a fast rate and with higher resolution than presently available. The final goal of the system will be capable of scanning a $30 \mathrm{~cm} \times 30 \mathrm{~cm} \mathrm{Nb}$ sheets and detecting $25 \mu \mathrm{m}$ Ta defects in less than 10 to 15 minutes. SQUID based detectors have a much higher sensitivity than normal eddy current systems. For this reason, AMAC, in collaboration with Christopher Newport University (CNU), Fermi National Accelerator Laboratory (FNAL), and Jefferson Laboratory (JLab), proposes to develop this system for fast scanning of $\mathrm{Nb}$ sheets with 4 times higher resolution than is currently available. The studies completed in Phase I have proved the feasibility of the project, realized the fast scanning \& data acquisition while the scanner is moving, and the preliminary data analysis system to identify the defects.

The development of such a system would be a step further in the development of this technology and would allow us to take a lead in the prototyping of a fast and reliable defect scanning technology.

In phase II we will prototype a working system, which will be capable of scanning a $30 \mathrm{~cm} \mathrm{x}$ $30 \mathrm{~cm} \mathrm{Nb}$ sheets and detecting Ta defects down to 25 microns in less than 15 minutes. The system will be further optimized, sensitivity will be improved and software will be enhanced so that an innovative 3-D SQUID based equipment for testing $\mathrm{Nb}$ sheets will be prototyped for DOE lab applications \& commercialization. Figure 1 is the old SQUID based system and the modification for the Phase I studies.

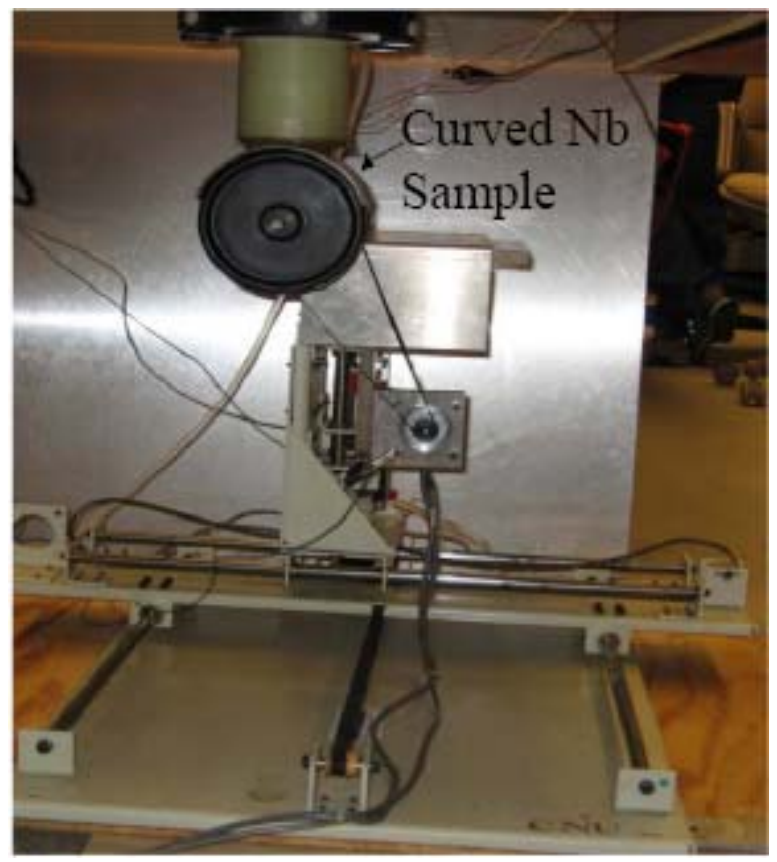




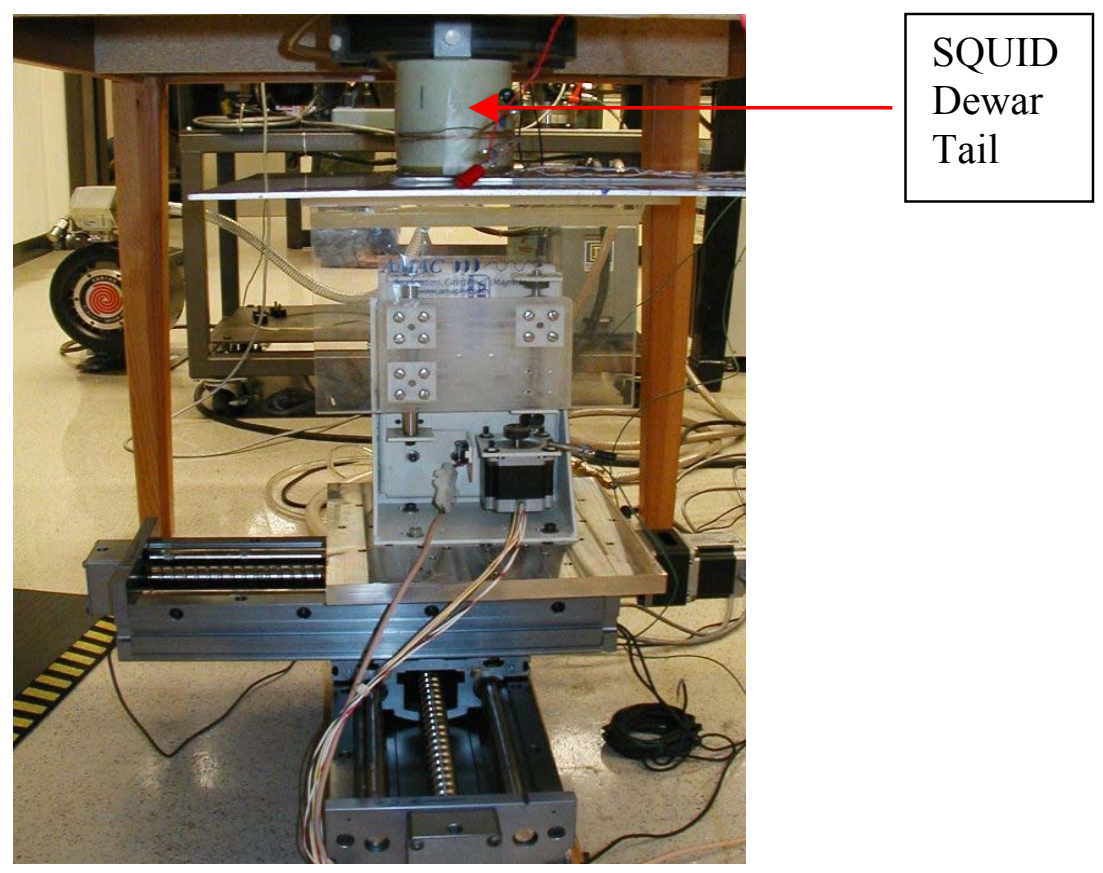

Fig. 1a) AMAC's preliminary designed SQUID system for scanning cylindrical curved samples. Fig. 1b) AMAC's preliminary prototype SQUID system aimed for fast scanning of flat $\mathrm{Nb}$ sheets (both work successfully for feasibility studies)

\section{B. TEAHNICAL APPROACH}

\section{B. 1. Technical Background}

The highest achievable accelerating field in Superconducting Radio frequency (SRF) cavities is proportional to the maximum surface magnetic field on the cavity wall, the so-called superheating critical field Bsh. The highest experimental field ever obtained on actual cavities made from bulk niobium materials exceeds $170 \mathrm{mT}$, very close to the estimated theoretical Bsh of $200 \mathrm{mT}$. Other superconducting materials exhibit lower critical fields while thin film technology suffers from high losses exhibiting a quality factor $\mathrm{Q}$ strongly degraded at high fields. To the state-of-the-art accelerating gradient Eacc of $25 \mathrm{MV} / \mathrm{m}$ in $\beta=1$ cavities (like those for electron accelerators) is relevant to $100 \mathrm{mT}$. Theoretically, $200 \mathrm{mT}$ is relevant to $45 \sim 50 \mathrm{MV} / \mathrm{m}$. It seems that one of the theoretical limit level is a rather hard magnetic barrier. To avoid this brick wall new cavity geometry is a possibility to boost Eacc. The trick is to alter cavity shape for a reduced Hpk/Eacc. Currently with new geometry, 10 15\% improvement in Eacc is possible (DESY, Jlab \& Cornell have done a great deal of work). A shape comparison is shown in Fig. 2. 


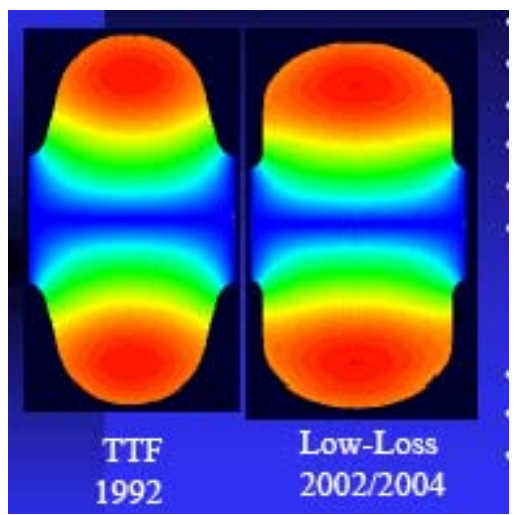

Fig. 2. Left is the Original DESY TTF SRF Cavity Shape. Right is the New Proposed Cavity Shape with Reduced Hpk/Eacc Values.

The observed quench fields of cavities is usually lower than the expected and calculated one assuming the uniform heating case by magnetic fields shown in Fig. 3. At the same time, a localized heating spot is detected by temperature mapping indicating that a thermal instability is occurring driven by a micron-size defect. More complete thermal analysis confirm that this is indeed the case and can explain why the quench changes with the heat properties of the helium bath.

Cavity performances are limited by electron loading (contamination), Q-drop (surface, interface, grain boundary and quench (defects, Hsh). Many attempts have been made over the years to correlate cavity performance and surface features. So far the most efficient diagnostic instrument is T-mapping. However, all these instruments are working only while cavities are working and/or cryogenic testing, i.e. cavities have been made. One has been looking for instruments, which will be able to quickly locate the micro defects (such as Ta inclosures in $\mathrm{Nb}$ sheet) in $\mathrm{Nb}$ sheets during and before fabrication.

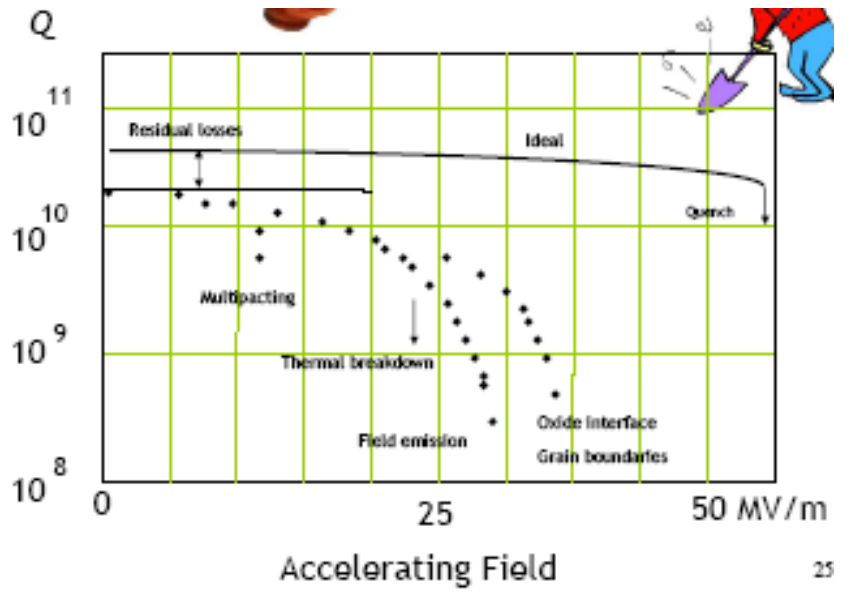

Fig. 3 A Comparison Among the Theoretical Performance (Upper) and Real Cavities Performance (Lower). 
SQUIDs are the most sensitive detector of magnetic flux and have unparallel sensitivity, bandwidth, spatial resolution, and fempto-tesla field resolution. Its working principles are shown in Fig. 4. Compared to conventional magnetic sensors, the SQUID has the capability of detecting magnetic signals that are many orders of magnitude smaller. As a result, the SQUID sensor can detect smaller defects than a conventional sensor if all other parameters are the same. The DC SQUID has the additional advantage of a flat response at low frequencies, which allows it to detect low frequency magnetic fields and allow a detection of inclusions deep into the materials. Superconducting Quantum Interference Devices (SQUID's) are the most sensitive magnetic sensors presently available and they can be used for high sensitivity magnetic field detection in eddy current based Non Destructive Testing (NDT) devices.

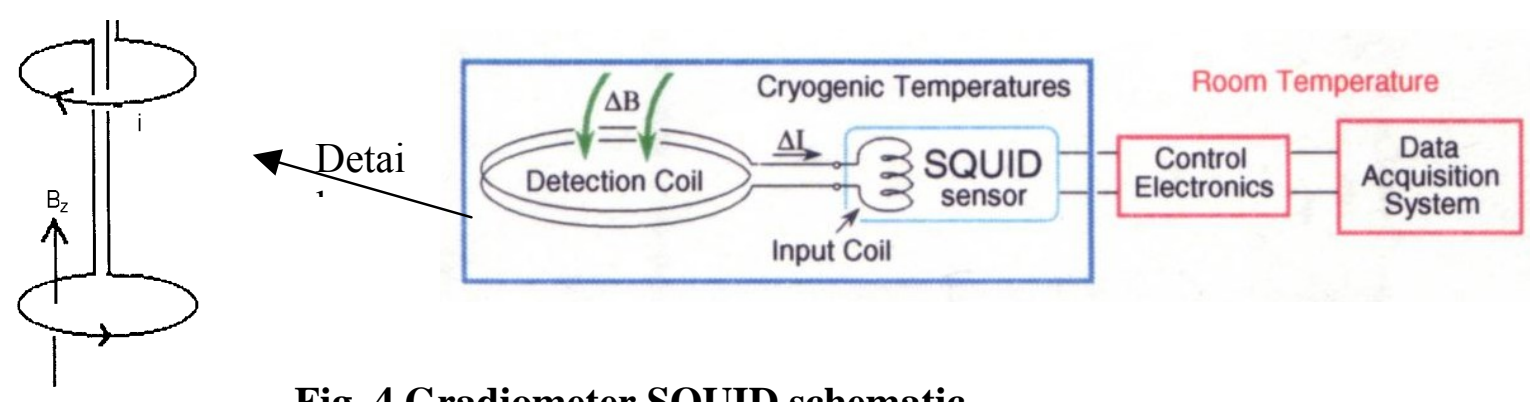

Fig. 4 Gradiometer SQUID schematic

\section{B. 2. Technical Approaches in the Phase I}

In Figure 5 is a schematic diagram of the SQUID system and its data acquisition in AMAC's Phase I studies. The system consists of a liquid Helium dewar, a SQUID probe and iMAG SQUID controller built by Tristan Technologies (San Diego, CA.) based on the specifications of our team. The SQUID output is connected to Stanford Research SR530 lock-in amplifier to isolate the signal produced from the induced current. The lock-in amplifier produces an inphase signal $(\varnothing=0)$ and a quadrature signal $(\varnothing=90)$. The system includes a 3-d motion controller that controls the motion of the sample under the tail of the dewar. The computer controls the motion system through a National Instruments PCI-7344 Motion Controller PC card. A Motor driver by National Instruments (MID-7604) powers a three axis setup: x, y, for translation, $\mathrm{z}$ for elevation.

The experiment is fully automated using LabVIEW software that allows the computer to interface with all the components of the system. The computer would allow the user to set the scanning step size (usually $1 \mathrm{~mm}$ ), parameters of the SQUID controller and the settings for the lock in amplifier. Figure 5, below, presents a schematic diagram of the SQUID setup that is used to scan $\mathrm{Nb}$ flat samples. The system operates by moving the sample below the pickup coil. Therefore, in our data, one pixel represents a scanned area of $1 \mathrm{~mm}^{2}$. Each file produced in scanning a sample contains two sets of data: in-phase $X(0)$, and quadrature $X(90)$.

The DC Low Temperature Superconductor (LTS) SQUID system includes a gradiometer probe and a non-magnetic liquid He dewar. The LTS type SQUID is selected for its higher field sensitivity $\left(\sim 50 \times 10^{-15}\right.$ Tesla). The SQUID gradiometer uses two coils wound in opposite 
directions that are configured as $\mathrm{dB}_{\mathrm{z}} / \mathrm{dz}$ gradiometer to cancel the effect of uniform magnetic fields as shown schematically in Fig.2. As a result, the SQUID gradiometer has the advantage of being able to be used in a non-magnetically shielded environment. The signal detected by the SQUID system is due to the net flux caused by the non-uniform magnetic field generated by the eddy current near the defect.

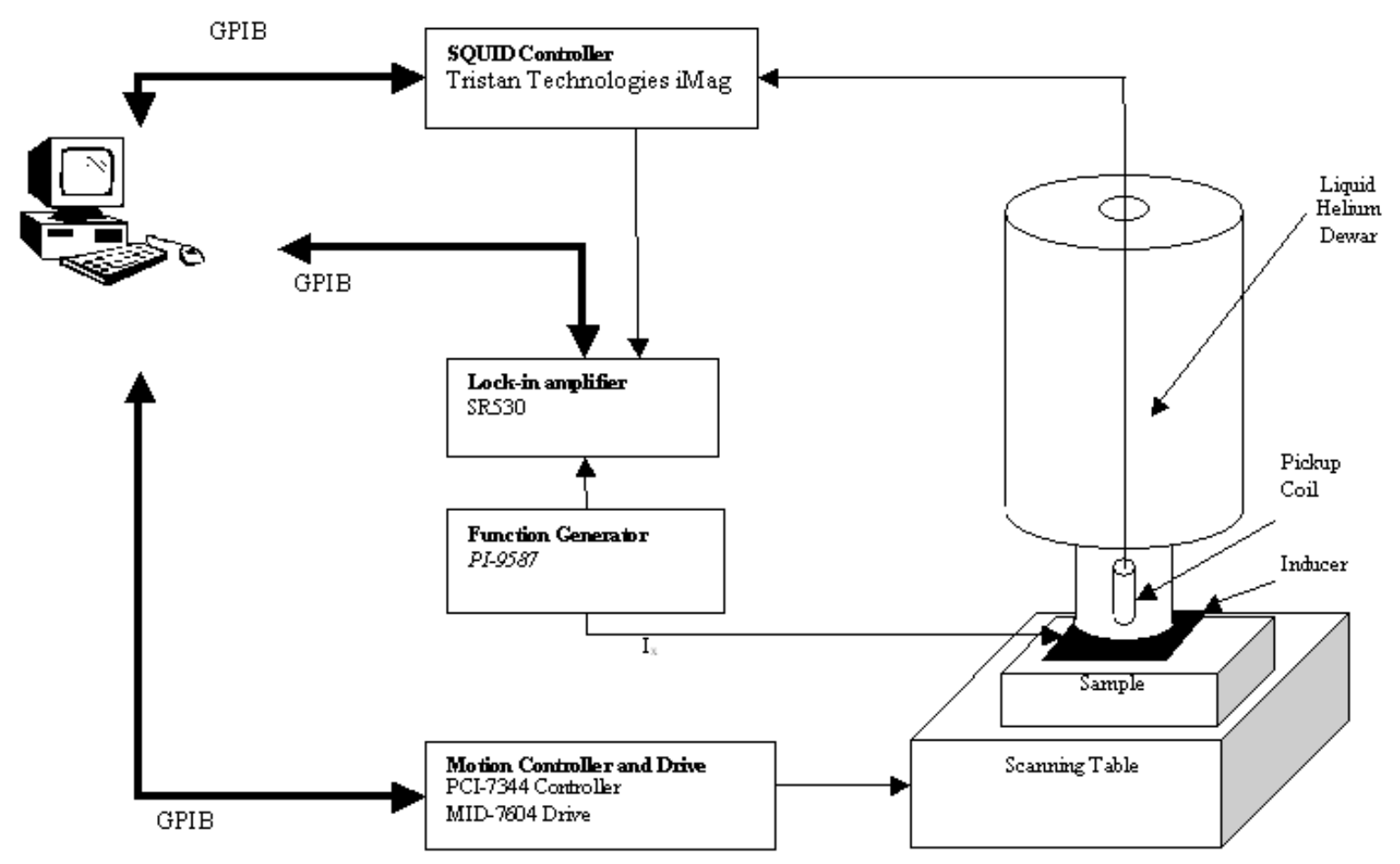

Fig. 5. A schematic diagram of the data acquisition system for low temperature SQUID system for scanning Nb sheets.

The system described above was successfully used in scanning $\mathrm{Nb}$ sheets and has resulted in detecting Ta inclusions that are 100 microns in diameter located on the surface, below the scanned surface or on the back side of the scanned sample. We were also able to detect these impurities by examining raw data files and without significant data analysis. We propose the following changes to upgrade the SQUID NDE system to improve its spatial resolution, to increase its scanning speed and to detect smaller impurities.

SQUIDs are the most sensitive detector of magnetic flux with unparallel sensitivity, bandwidth, spatial resolution, and fempto-tesla field resolution. Compared to conventional magnetic sensors, the SQUID has the capability of detecting magnetic signals that are many orders of magnitude smaller. As a result, the SQUID sensor can detect smaller defects than a conventional sensor, if all other parameters are the same. The DC SQUID has the additional 
advantage of having a flat response at low frequencies, which allows it to detect low frequency magnetic fields (as low as few hertz). Eddy current techniques that use conventional magnetic sensors have the disadvantage of using high frequencies that are useful in detecting surface defects. Eddy current systems that use SQUID sensors have the advantage of using low frequencies for the excitation currents which allows the system to detect flaws deep into the material [3]. This can be explained by the fact that the generated eddy current is in general at the surface of the material and penetrates the material only down to small distance defined as the skin depth or the penetration depth. The penetration depth $(\delta)$ depends on the conductivity $(\sigma)$ of the material as well as the frequency $(\omega)$ of the excitation current according to the formula: $\delta=c / \sqrt{ }(2 \pi \mu \sigma \omega)$

If one uses a high frequency excitation current as in the case of conventional eddy current methods, then the skin depth is small and the eddy current is at the surface and as such can detect only surface defects. A low frequency excitation current will have a larger skin depth, allowing the possibility of detecting deep defects. As a result detecting deeper defects becomes very challenging. The high field-sensitivity of DC SQUID sensors, especially at low frequencies, allows us to detect such small signals. Based on the skin depth parameters for $\mathrm{Nb}$, the optimal frequency for scanning defects at surface is $100 \mathrm{KHz}$. For defects $1 \mathrm{~mm}$ from the surface the optimum frequency is $40 \mathrm{KHz}$, and for defects that are $2 \mathrm{~mm}$ deep, one needs to use a frequency of $10 \mathrm{KHz}$.

The use of DC SQUID sensors in nondestructive evaluation, specifically for detecting defects in metals has been well documented. In particular, eddy current techniques have been used in SQUID systems to image defects deep under the surface using relatively low frequencies. The use of such a SQUID system in detecting Ta inclusion in NB sheets and cavities will enhance the capabilities of the system in terms of increased sensitivity and spatial resolution.

Previously we managed to detect these small Ta inclusions using a SQUID scanning system [5], but the scanning time was too long. In fact it took few hours to scan a $30 \mathrm{~cm} \mathrm{x} 30 \mathrm{~cm} \mathrm{Nb}$ sheet with a step of $1 \mathrm{~mm}$. The previous system operated by moving the sample a distance of 1 $\mathrm{mm}$ then a measurement by the SQUID is activated after a short settle time. This settle time of 1-2 seconds was important to allow the mechanical vibrations to dampen as well as the electromagnetic fields of the motor to diminish. As a result this produced a long scanning time due to the fact that we needed to stop and wait for transient effects to settle before activating the SQUID measurement. To speed the scanning process, we needed to scan in a continuous mode without stopping at $1 \mathrm{~mm}$ intervals to take readings. We proposed to design and implement a SQUID scanning system that is fast and is capable of scanning a $30 \mathrm{~cm} \times 30 \mathrm{~cm}$ $\mathrm{Nb}$ sheet in 15 minutes by continuously moving the table at speeds up to $10 \mathrm{~mm} / \mathrm{s}$ while activating the SQUID at $1 \mathrm{~mm}$ interval.

\section{ANTICIPATED PUBLIC BENEFITS}

1) The purposed SQUID based NDT system will ultimately help reach the highest possible accelerating field in cavities and lower the cost of new accelerators as well as the cost for upgrading existing accelerators. The capability of detecting Ta inclusions down to $25-\mu \mathrm{m}$ size will allow the production of superconducting 
RF-cavities with higher electric field limits, and reduce the fabrication cost by eliminating defective sheets prior to their use in fabricating the SRF cavities. The increase in accelerating field also affects the cost of the accelerator by reducing the length required for the SRF cavities, or by allowing the installation of higher gradient cavities for energy upgrades occupying the same space as the lower gradient cavities in existing machines. This will produce a considerable cost reduction for new accelerators as well as for upgrades of existing accelerators using SRF cavities, since the savings in cavities structure length impact the cost of many auxiliary systems as well as the accelerator tunnel length.

2) The SQUID based scanning system could also be used in other applications to detect defects in metallic structures (magnetic and non-magnetic impurities), and in materials that are of interest to NASA, the Air Force and other National DOE Laboratories.

\section{Phase I TECHNICAL OBJECTIVES}

The AMAC/CNU team with support from FNAL/BNL/JLab will develop a SQUIDbased eddy current NDT system to detect impurities in $\mathrm{Nb}$ sheets that are used in manufacturing of SRF cavities. The proposed system needs to be developed to achieve higher sensitivity and faster scanning rates over existing systems.

The first objective is the Phase-I work to detect Ta impurities smaller than $50 \mu \mathrm{m}$ in diameter. This will be accomplished by reducing size of the detection coil for the SQUID system to $1 \mathrm{~mm}$. The second objective of Phase-I is to design and develop the scanning system for a continuous scanning of the $\mathrm{Nb}$ sheets. The Phase-I development will include hardware design and implementation of the SQUID pick up coils, software development for fast scanning and further development of data analysis tools. The system needs to be tested using $\mathrm{Nb}$ sheets with inclusion samples specifically fabricated for this task. The tests will verify the proper functioning of the system and set the parameters for the PhaseII program.

The success in the phase I project will pave a solid ground for the phase II project in development, optimization, and prototype of the SQUID-based detection system. The system will allow the production of superconducting RF-cavities with higher electric field limits, and reduce the fabrication cost by eliminating defective sheets prior to their use in fabricating the SRF cavities.

\section{E. PHASE I PERFORMANCE SCHEDULE}

The Phase-I work plan is the guide to reach the Phase-I technical objectives, and the results of the investigation will be used for the preparation for the Phase-II program. The tasks for Phase-I can be broken down as follows: 


\begin{tabular}{|l|l|l|l|l|l|l|l|}
\hline & Task & $\begin{array}{l}\text { Mont } \\
\text { h 1 }\end{array}$ & $\begin{array}{l}\text { Month } \\
2\end{array}$ & $\begin{array}{l}\text { Mont } \\
\mathrm{h} \mathrm{3}\end{array}$ & $\begin{array}{l}\text { Month } \\
4\end{array}$ & $\begin{array}{l}\text { Mont } \\
\mathrm{h} \mathrm{5}\end{array}$ & $\begin{array}{l}\text { Month } \\
6\end{array}$ \\
\hline 1 & $\begin{array}{l}\text { Design and modification of the } \\
\text { SQUID probe and the detection coil }\end{array}$ & & & & & & \\
\hline 2 & $\begin{array}{l}\text { Implement hardware and software } \\
\text { changes for continuous scanning of } \\
\text { Nb plates }\end{array}$ & & & & & & \\
\hline 3 & $\begin{array}{l}\text { Fabrication of Niobium sheet } \\
\text { samples with known defects and } \\
\text { inclusions }\end{array}$ & & & & & & \\
\hline 4 & $\begin{array}{l}\text { Perform test scans of the sample } \\
\text { Niobium sheets }\end{array}$ & & & & & & \\
\hline 5 & \begin{tabular}{l} 
Enhance the data analysis software. \\
\cline { 5 - 7 }
\end{tabular} & & & & & & \\
\hline 6 & $\begin{array}{l}\text { Analyze results and develop Phase- } \\
\text { II plan, }\end{array}$ & & & & & $\longrightarrow$ \\
\hline 7 & Write final report to DOE & & & & & & $\longrightarrow$ \\
\hline
\end{tabular}

For the Phase-II, the sensitivity will be further improved to detect defects down to 25 microns by locating the detection coil of the SQUID system outside the Helium dewar. This modification to the design of the SQUID system is more elaborate and more expensive to be considered in phase I. The sample support system will also be modified and will be mounted on a robotic system to reduce the lift-off effects. In phase II, we will upgrade the SQUID system by using a linear array of two SQUID sensors with two pick up coils, effectively scanning 2 lines with each sweep. Using the 2-SQUID sensor system will allow us to scan the same $30 \mathrm{cmX} 30 \mathrm{~cm} \mathrm{Nb}$ sheet in half the time i.e. in 7.5 minutes. Finally, a completed prototype will be constructed and optimized for future commercialization.

\section{F. RELATED RESEARCH OR R\&D}

A method of scanning the high purity niobium sheets as supplied by the manufacturer has been developed at DESY for the TTF cavities by using the eddy current method. The eddy current method has one intrinsic set back: the resolution of the method is limited to defects of a size not smaller than 100 micron; the limitation in resolution is resulting from the finite dimensions of the excitation and detector coils in the eddy current sensor. Therefore, if one is interested in detecting defects of much smaller sizes, the elimination of which are necessary in achieving higher gradients, a method with higher resolution must be employed. Such higher resolutions can be achieved with SQUID-based detection systems. The new DESY SQUID scanner now typically achieves a resolution of about 100 micron tantalum particles on flat $\mathrm{N}_{\mathrm{b}}$ sheets.

The development of an improved system would be a step further in the technology and would allow AMAC/BNL/CNU/FNAL to take a strong lead in the development of 
the defect scanning technology required for the fabrication of superconducting cavities and other applications. The AMAC team has been involved in the detection of impurities in aluminum structures as well as $\mathrm{Nb}$ sheets using eddy current SQUID systems. As pointed out earlier, we were able to clearly detect 100 microns Ta particles on the surface and on the back side of $\mathrm{Nb}$ sheets.

\section{G. PHASE I R\&D and ACCOMPLISHMENTS}

\section{G. 1. Experimental Set up and the Data Acquisition System}

We have designed and constructed a DC low temperature SQUID system for this project that is capable of scanning flat NB samples. The Dewar, SQUID probe and iMAG controller was built by Tristan Technologies (San Diego, CA.) based on the specifications of our team. The SQUID output is connected to Stanford Research SR530 lock-in amplifier to isolate the signal produced from the induced current. The lock-in amplifier produces an in-phase signal $(\varnothing=0)$ and a quadrature signal $(\varnothing=90)$. The system includes a 3-d motion controller that controls the motion of the sample under the tail of the dewar. The computer controls the motion system through a National Instruments PCI7344 Motion Controller PC card. A Motor driver by National Instruments (MID-7604) powers a three axis setup: $\mathrm{x}, \mathrm{y}$, for translation, $\mathrm{z}$ for elevation. We have installed a new scanning table which uses a screw mechanism to greatly reduce vibrations and allow us to scan in continuous fashion.

We have also developed fully automated measurement system using LabVIEW software that allows the computer to interface with all the components of the system. This custom data acquisition system allows for choosing the scanning speed in the range from 0.1 $\mathrm{mm} / \mathrm{s}$ to $10 \mathrm{~mm}$, update the position of the scanning table activate a measurement at uniform intervals of $1 \mathrm{~mm}$. Therefore in our data, one pixel represents a scanned area of 1 $\mathrm{mm}^{2}$. Each file produced in scanning a sample contains two sets of data: in-phase $\mathrm{X}(0)$ and quadrature X (90). The parameters for the SQUID controller and the settings for the lock in amplifier are also controlled by the Labview software.

Figure 5 (section B) presents a schematic diagram of the SQUID setup and Figure 6 shows a photograph of the setup that is used to scan flat samples. Figure 7 is the data acquisition electronics. 
AMAC Technical Closeout Report - "High Resolution and Fast Scanning Speed SQUID

Based Non-destructive Inspection System of Niobium Sheets for SRF Cavities"

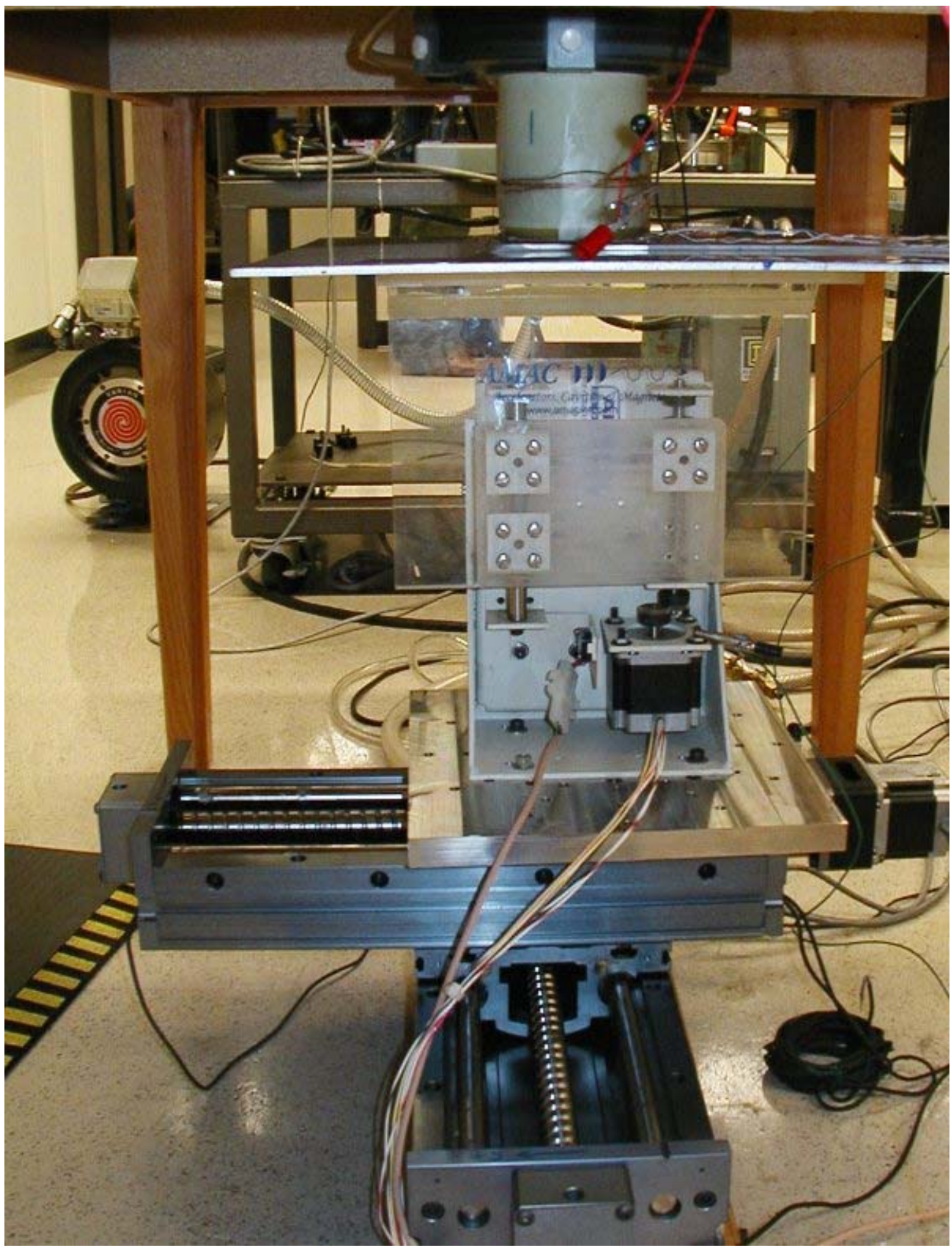

Figure 6: The Low Temperature Squid System showing the scanning of a $30 \times 30$ cm Nb sheet 


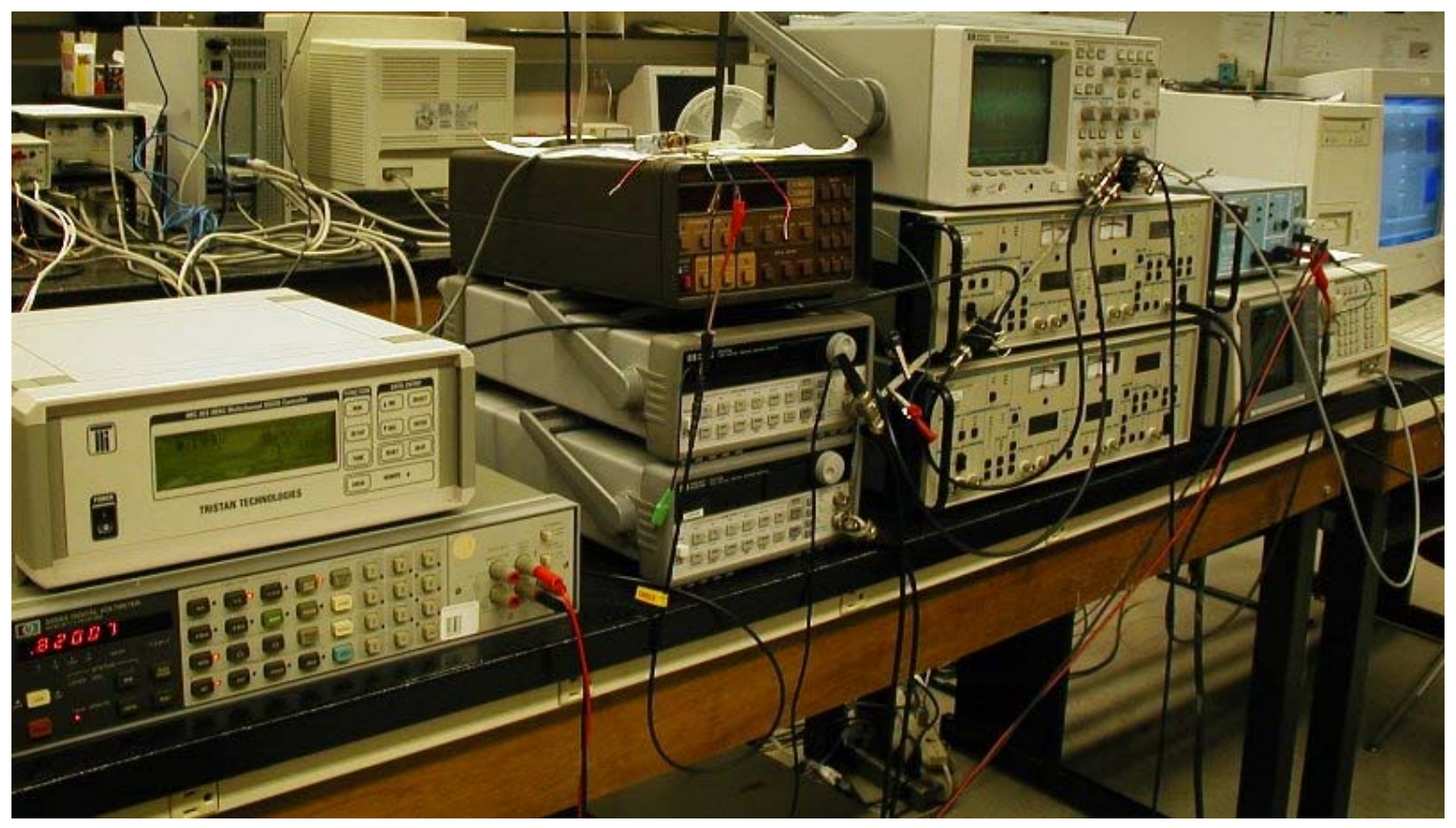

Figure 7: Data Acquisition Electronics

The Dewar is constructed of fiberglass and contains a collapsible tail apparatus to adjust the distance to the sample. To increase sensitivity, the distance between the bottom of the Dewar tail and the pickup coil can be adjusted to a minimum of $2 \mathrm{~mm}$ during operation. The gradiometer probe has a pick up coil that is configured as $\mathrm{dB}_{\mathrm{Z}} / \mathrm{dz}$ gradiometer to cancel the effect of uniform magnetic fields from the environment. To increase the spatial resolution the pickup coil is required to be as small as possible. The pickup coil with our system has a diameter of $2 \mathrm{~mm}$, the smallest possible with current wire winding technology. Figure 8 shows the SQUID probe for our SQUID system. Although the spatial resolution of the system is determined by the size of the pickup coil, we have used a large excitation current $(0.5-1.0 \mathrm{~A})$ through the inducer to increase the magnitude of the eddy current in the sample which increases the signal produced by the defect and consequently enhance the possibility of detecting smaller defects. The overall sensitivity of this system is $\sim 1.5 \times 10^{-7}$ Tesla $/$ Volt when using the most sensitive scale of the SQUID controller. 

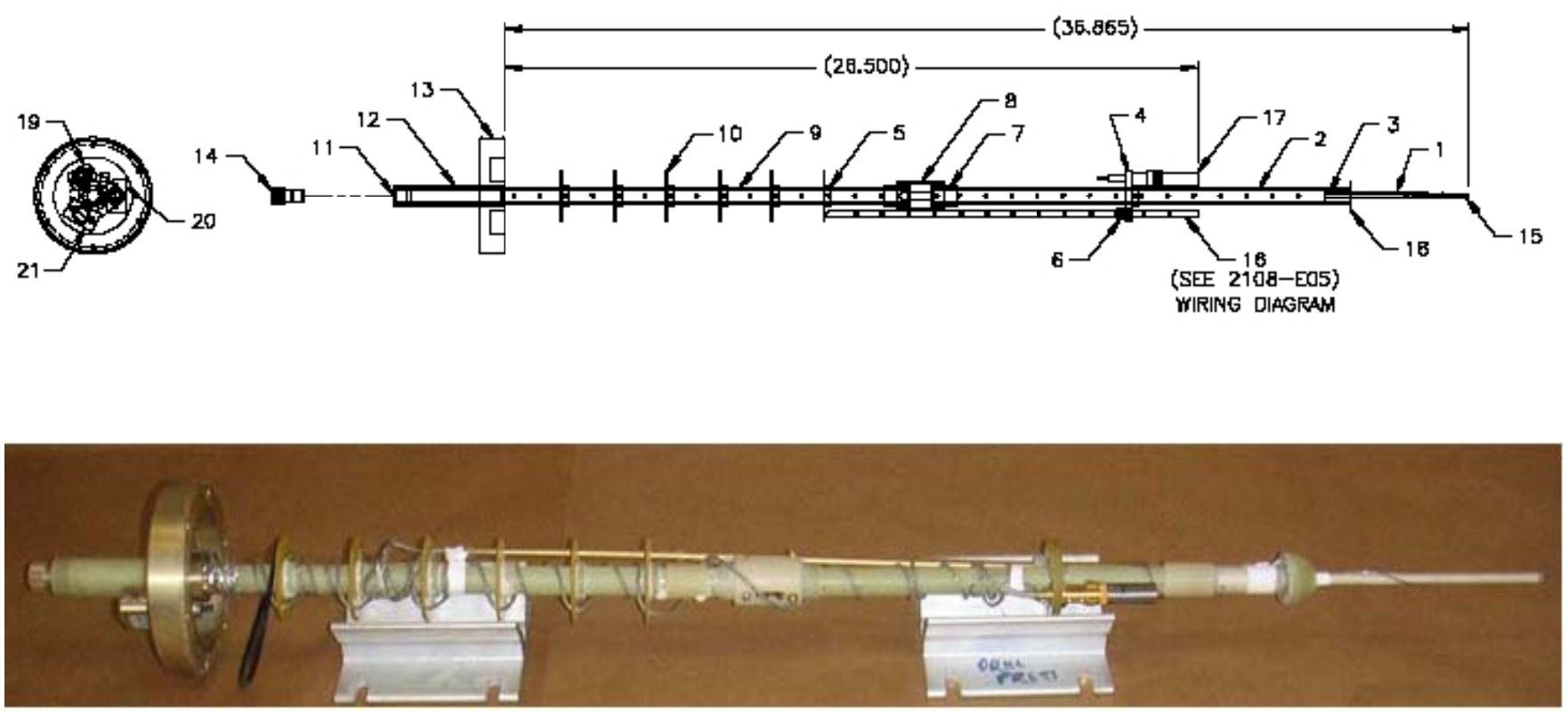

\section{Figure 8: SQUID probe}

We have used a planar current inducer fabricated from copper PC board $(8.1 \mathrm{~cm}$ long $\mathrm{x}$ $8.1 \mathrm{~cm}$ wide) excited by a Pasco Scientific PI-9587 Function Generator/Amplifier. The advantage of this planar current inducer is that it produces a more uniform eddy current in the sample, makes easier to initially "zero off the SQUID signal when no defect is present and as a result enhances the chances of detecting the smaller signal of the defect.

The signal detected by the SQUID is due to the net flux threading the pick up coil. A defect will cause an alteration in the current. From Faraday's Law,

$$
\begin{aligned}
& \nabla \times E+\frac{d B}{d t}=0 \\
& E=\frac{d(I R)}{d x}
\end{aligned}
$$

any obstruction of this current causes change in the electric and magnetic field. Relative to the pick up coil, "a maximum" will be on one side of the defect and "a minimum" on the other. Figure 9 shows a typical result of a field detected by such a system. Here the current is in the $\mathrm{X}$ direction, the white indicating the maximum, black the minimum, and blue is the background. 


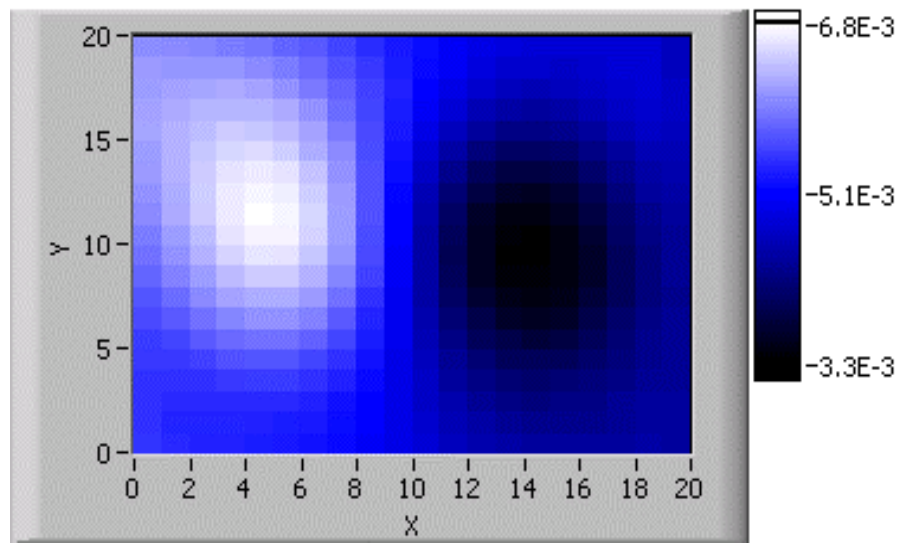

a)

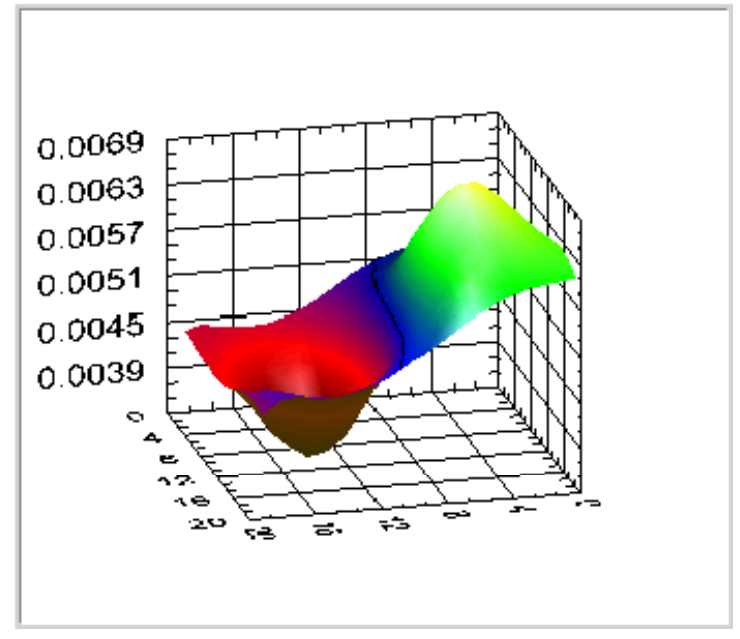

b)

Figure 9: a) Intensity chart shows the "maximum" and the "minimum" detected by a SQUID and produced by an eddy current in sample with a defect. b) $3 \mathrm{D}$ graph of the same results.

\section{G. 2. Data Acquisition Software}

We have developed custom software that uses LabView to implement the new design for the data acquisition of the SQUID system. We have added the capability of running the scanning table continuously and taking data without stopping. We have developed a VI that allows the scanning table to continuously run, control the velocity of each axis and output the $\mathrm{X}, \mathrm{Y}$, and $\mathrm{Z}$ positions of the sample while scanning. Therefore, some measurements and calculations had to be made to calculate the conversion factors for the encoder to calculate the position for each axis. For the hybrid stepper motor that was used, 1 rev $=2000$ encoder counts $=200$ motor steps. $1 \mathrm{RPM}=200 \mathrm{steps} / 60 \mathrm{~s}=3.33$ steps $/ \mathrm{s}=33.3$ counts $/ \mathrm{s}$, which translates into $=(33.3 / 200) \mathrm{mm} / \mathrm{s}$, hence $1 \mathrm{RPM}=0.166$ $\mathrm{mm} / \mathrm{s}$. We would trigger a measurement of the SQUID system when the scanning table is 
within $+/-.05 \mathrm{~mm}$ of the target position. Also, to improve our data, an average function was added to average all the readings of Lock-in amplifier collected at each point (in the range of $0.10 \mathrm{~mm}$ ). The average was a significant improvement since it smoothed out the output data by taking a statistical average of multiple readings. An overshoot at the beginning and at the end of each line scan had to be added, so accurate results will be collected on the first and last point. The overshoot was chosen to be $3 \mathrm{~mm}$ based on the overload time of the Lock-in amplifier. We also implemented a STOP option in order to stop the scan after completing the current line. All the data collected was saved on a text file, which has path specified by the user at the beginning of the program. Figure 10 is the front panel of the main VI.

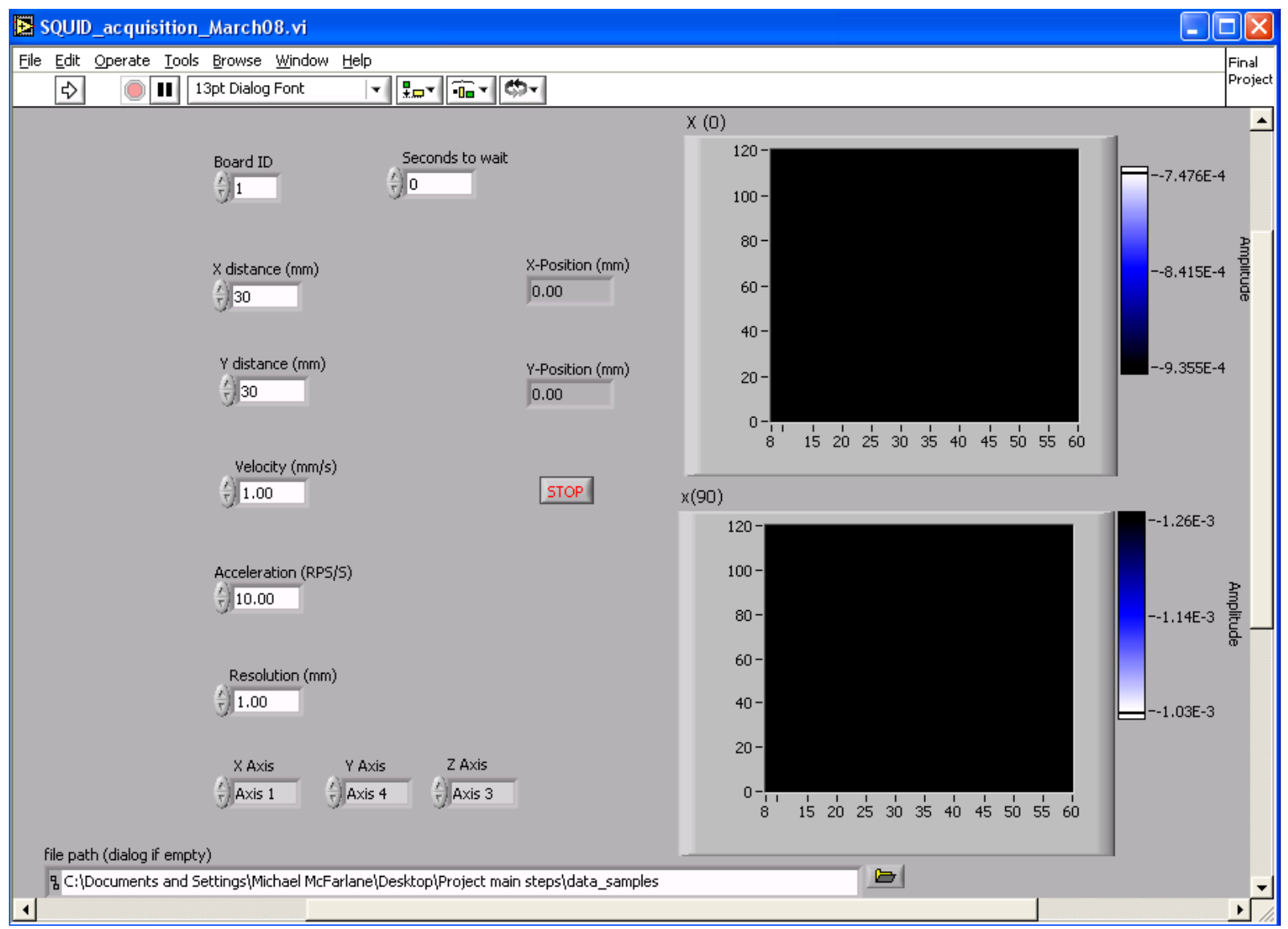

Figure 10: Front panel of the Main VI, SQUID Continuous Scanning 


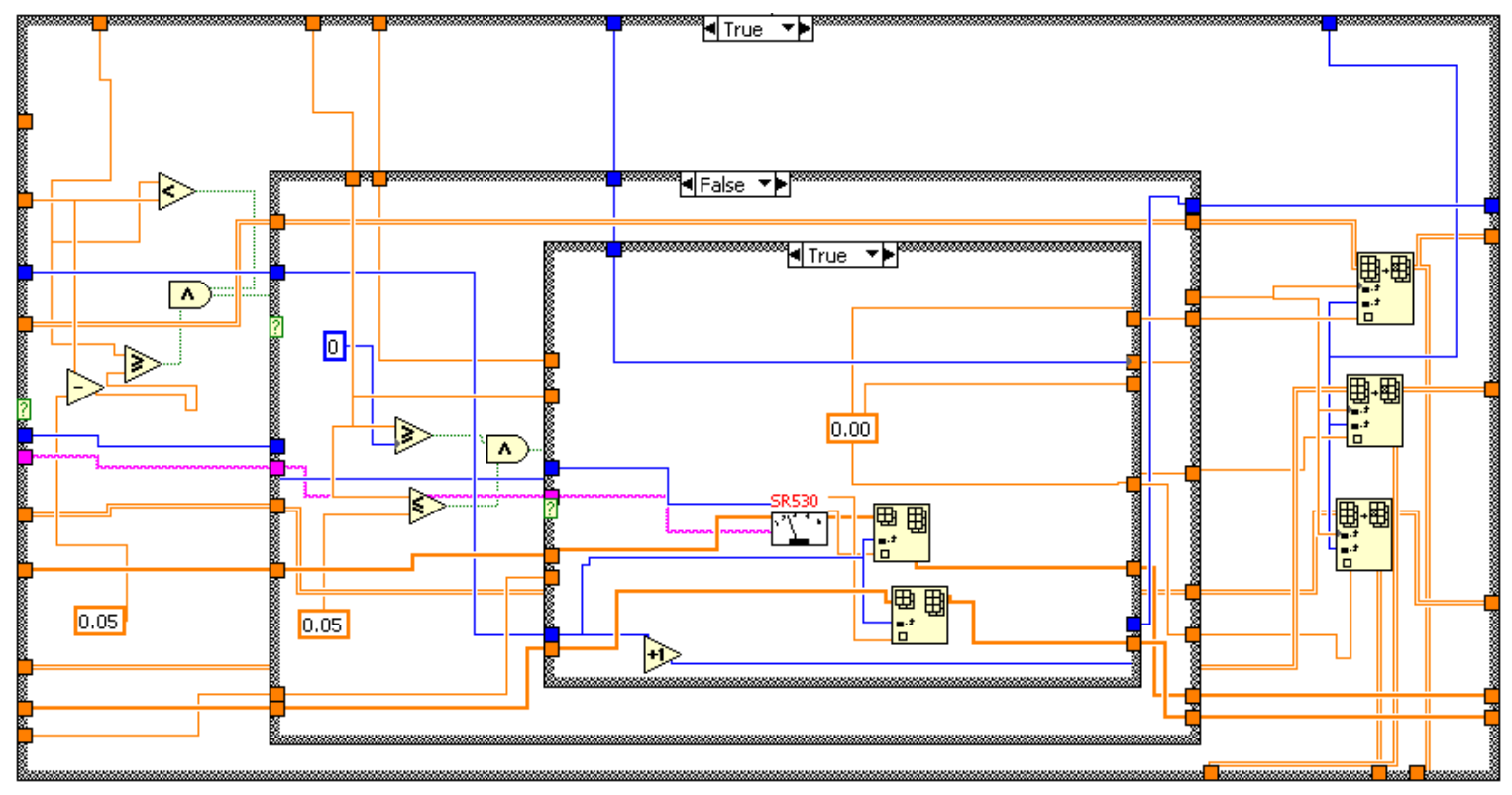

Figure 11: Wiring Diagram of reading data in a certain range.

We can see from the above Figure 11 diagram how does the VI collect the data triggered by our specified range. When the position is less than $X-0.05$ or higher than $X+0.05$, then no data is collected. Otherwise, when the desirable range is met, the VI reads the data from the Lock-in amplifier and inserts it in an array. This is one example of a portion that has been added to meet the specifications of the scan.

In addition to the main VI we have added two VIs for initialization of the scan parameters. The first VI was the initialization of the location of starting point, which allows the user to move the scanning table in the axis, $\mathrm{X}, \mathrm{Y}$ and $\mathrm{Z}$ to a certain initial position. Therefore, during the scan, the $\mathrm{X}$ and $\mathrm{Y}$ axis positions are incremented from that initial position. The program allows the user as Figure 12 and figure 13 to keep track of the $\mathrm{X}, \mathrm{Y}$ positions through the whole scan, and makes it possible to return to the origin position after the scan is completed. 


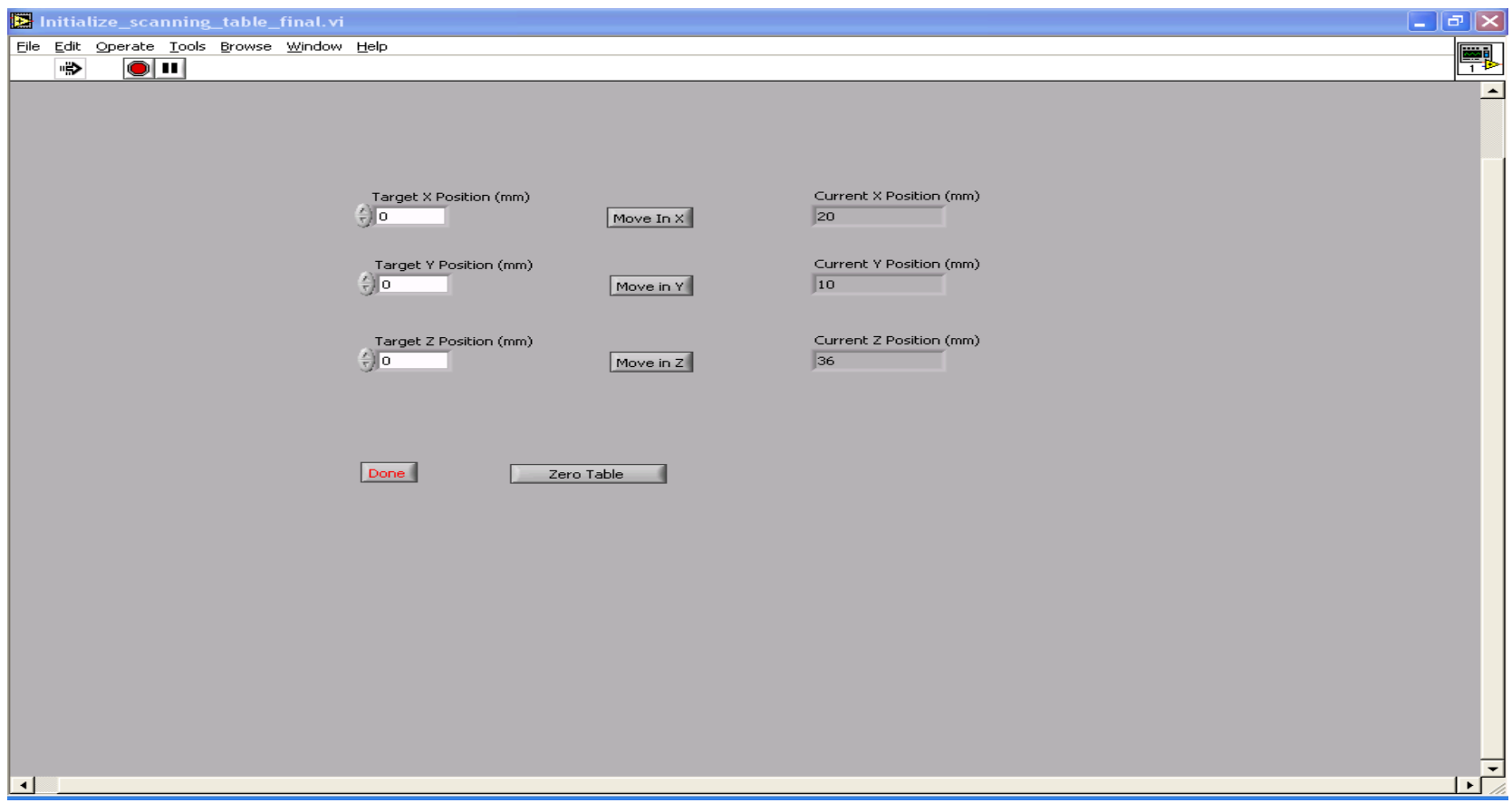

\section{Figure 12. Front Panel of Position Initialization}

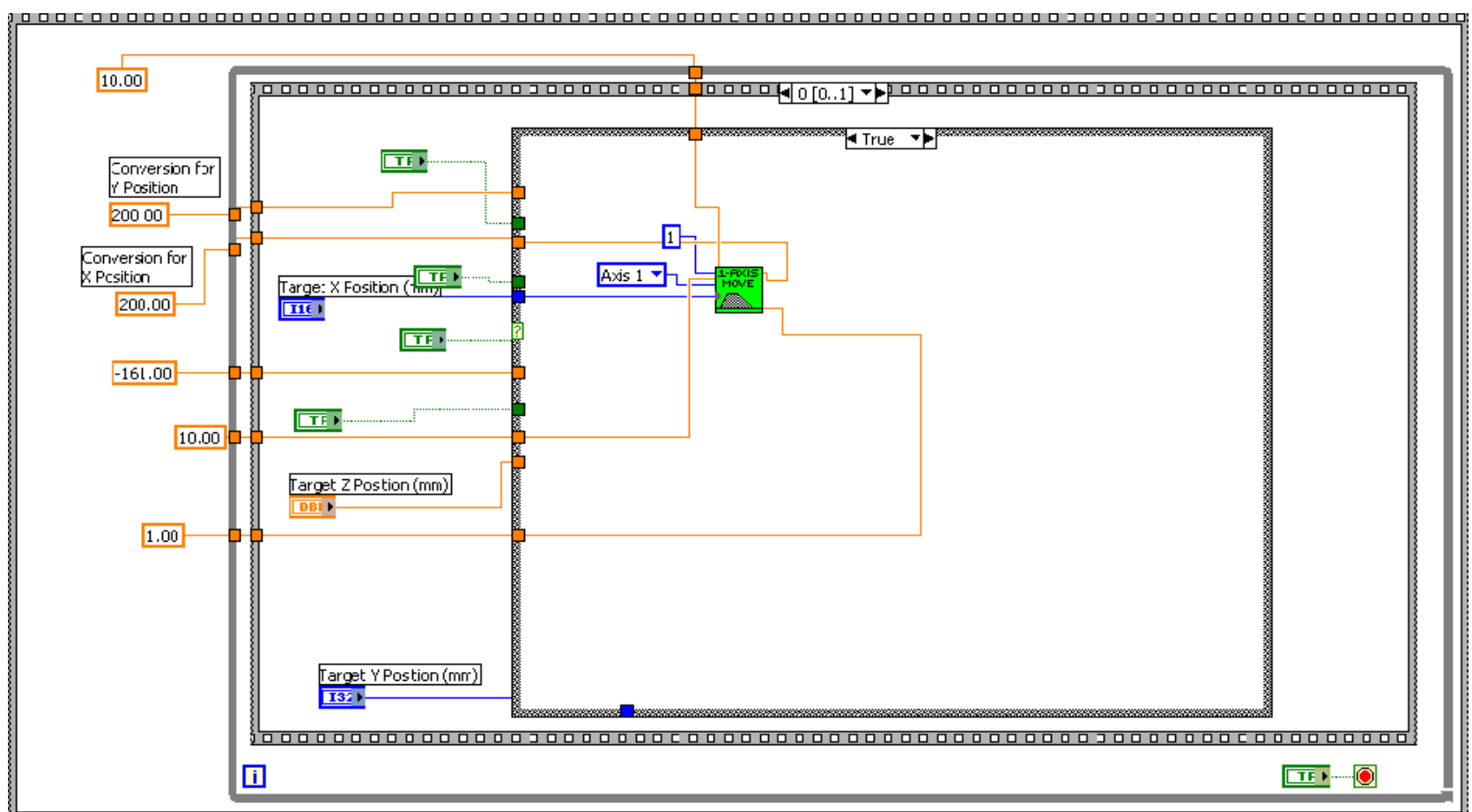

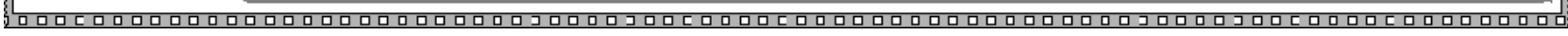

\section{Figure 13: Wiring Diagram of Position Initialization}


The above wiring diagram shows the conversion factors of the $\mathrm{X}, \mathrm{Y}$, and $\mathrm{Z}$ axis that have been used, which are 200, 200, -161 respectively. Also, it represents how the inputs are inserted.

The second initialization VI is for the SQUID control. The user is asked to choose the SQUID parameters: overload voltage, gain, low pass filter and high pass filter. After setting up these the parameters, the SQUID controller is set to those parameters. At the start of every line during the scan the SQUID is reset to ensure proper readings as Figure 14. After a reset, the SQUID needs some time to recover; a wait variable was implemented on the main VI to wait after the SQUID is reset.

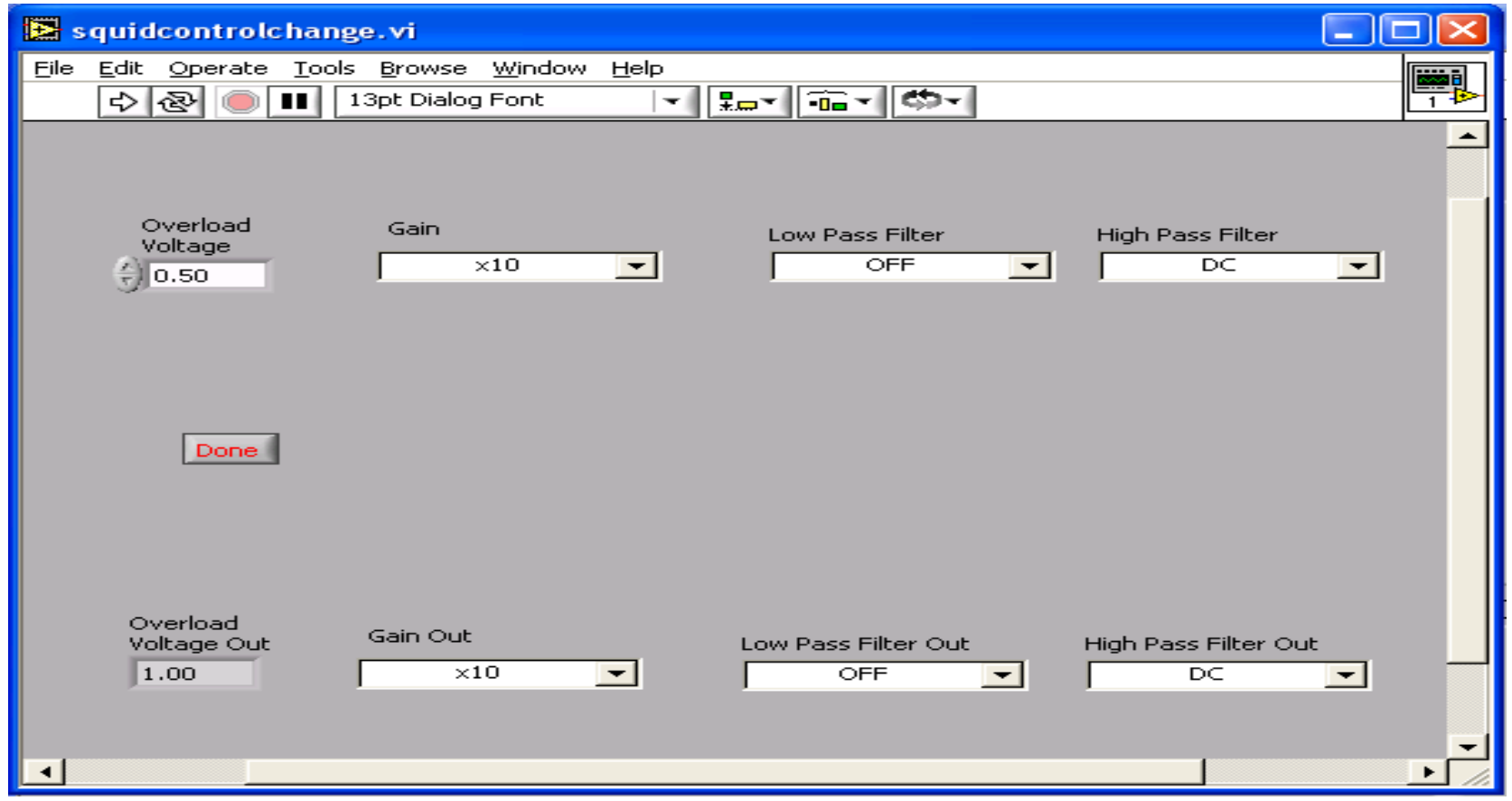

Figure 14. Front Panel of SQUID Reset Parameters 


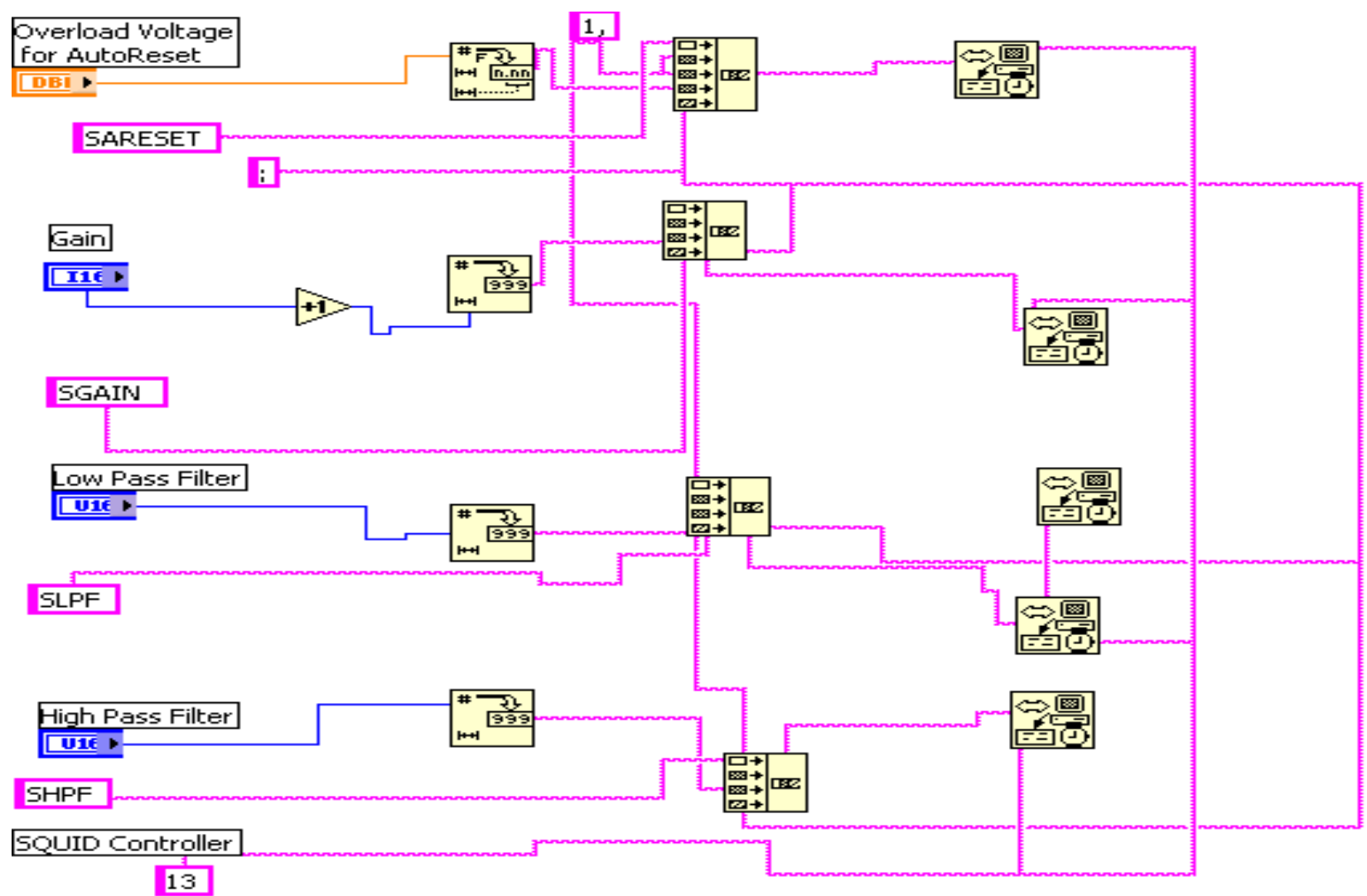

Figure 15: Wiring Diagram for setting SQUID Parameters

The above wiring diagram, Figure 15 shows how the desired parameters are passed to the SQUID controller. This is done using a GPIB function and some other basic functions such as, Number to Decimal string, which converts number to a string, and concatenate strings, which concatenate input strings into one output string. The GPIB function used on this SubVI is GPIB write, which writes our inputted parameters to the GPIB device identified by address 13 , which the SQUID controller.

\section{G. 3. DATA Analysis Software}

The lift off effect is one of the issues to needs to be dealt with in such scanning systems. The gap between the inducer and the scanned sample is not even and this will affect the magnitude of the signal detected by the SQUID. In order to deal with this issue, we developed a new filtering program to help in correcting the effect of having an uneven gap between the inducer and the scanned sample. Using the a polynomial fit function we were able to fit the collected data for each line and get the polynomial coefficients, which were used to get the derivative of that line. The general form of a polynomial function is: $\mathrm{F}(\mathrm{x})=\mathrm{a}_{\mathrm{n}} \mathrm{x}^{\mathrm{n}}+\mathrm{a}_{\mathrm{n}-1} \mathrm{x}^{\mathrm{n}-1}+\cdots+\mathrm{a}_{1} \mathrm{x}+\mathrm{a}_{0}$, where $\mathrm{a}_{\mathrm{i}}$ represents the polynomial coefficient, $\mathrm{n}$ is the polynomial order. In our case, the input $x$ values represent the position and the input $y$ values represent the corresponding reading by the SQUID. After getting the polynomial coefficients, the derivative of $\mathrm{F}(\mathrm{x})$, can be written as: 
$d F(x) / d x=a_{n} x^{n-1}+a_{n-1} x^{n-2}+\cdots+a_{2} x+a_{1}$. We were able to calculate the values of this derivative for every value $x$ of the scanned line. The VI repeats the same method for all data lines collected (horizontally) and outputs the new graph. In effect we were able to get rid of any slope (linear effect) that is produced by the lift off effect. The filtering was done first on the $\mathrm{X}$ axis and later on the $\mathrm{Y}$ axis (vertically). The filtering in the $\mathrm{X}$ and $\mathrm{Y}$ axis worked fine but in many cases it did not help much with our collected data. While working with this filter, it was challenging to figure out the right polynomial order to use and its effect on the data.

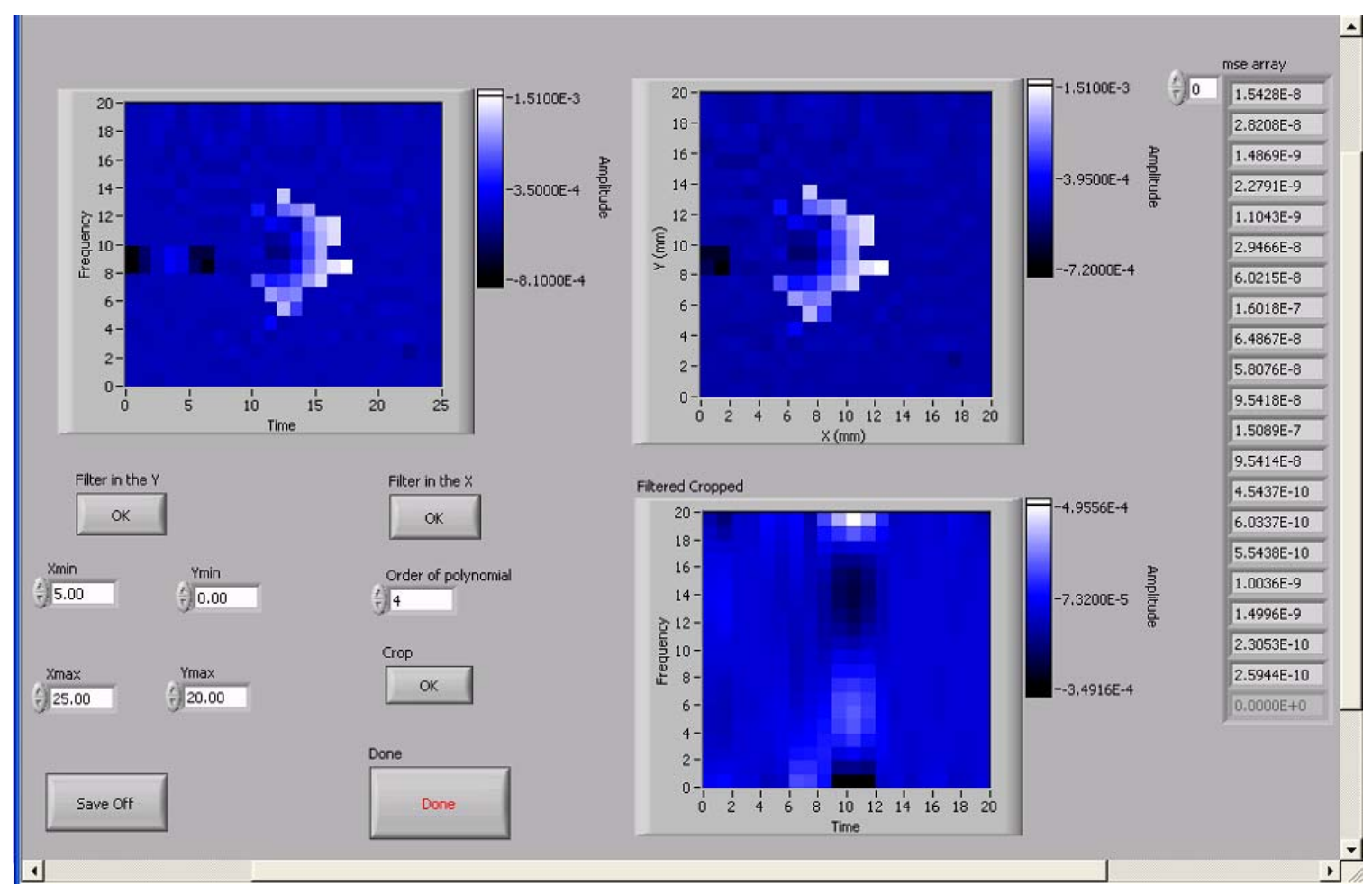

\section{Figure 16: Front Panel of the Filtering using Polynomial Fit}

On the front panel Figure 16, the user can enter all the inputs for the cropped graph and the order of the polynomial. The three graphs shown on the front panel show the origin graph of collected data (top left), the cropped area of the graph (top right), and the filtered graph (bottom). Once the program is in running mode, a load file window is displayed to load the data file. The order of polynomial has a significant effect on the filtering of the data, so the user may choose the proper order of polynomial by looking at the change of the filtered graph. The array on the right represents the mean squared error, which is calculated from the polynomial fit function. Also, there is a Save push button that allows to save the filtered cropped data and a Done push button to stop the run. 


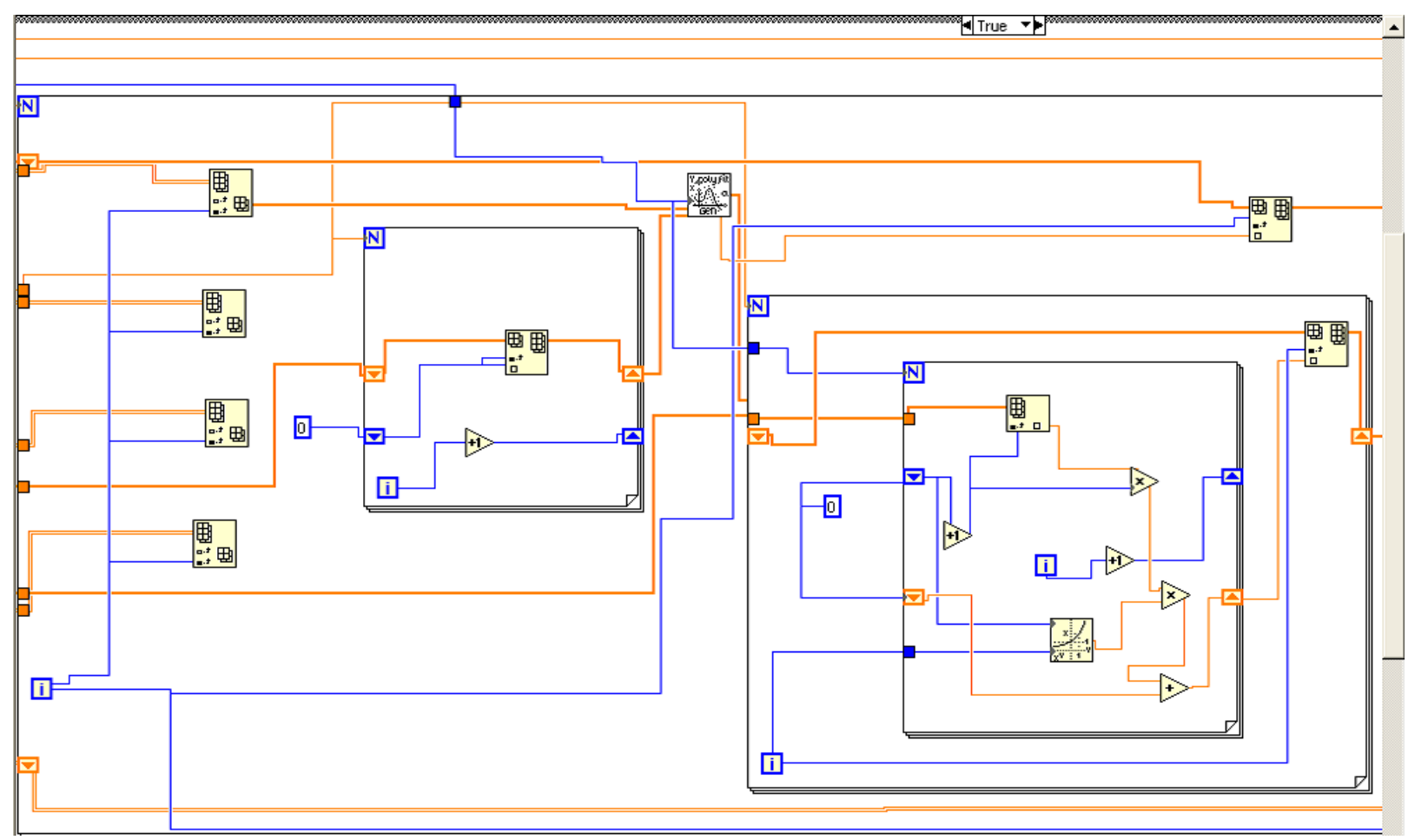

Figure 17: Wiring Diagram of the Filtering using Polynomial Fit

In the above wiring diagram Figure 17, we can see the two main parts of the filtering that have been used. The first part is input the X, Y values and the polynomial order to the Polynomial fit function, which returns the polynomial coefficients and the mean squared error. Then, the output coefficients are used to calculate the value of the derivative at each position $\mathrm{x}$. The result of each data point is stored in a an array, which is outputted on the graph of the front panel.

\section{G. 4. Results}

\section{1) Flat Nb sheet with Ta inclusions Made by AMAC:}

The next step is testing the system's ability to detect inclusions within niobium. Our first niobium sample with inclusions consisted of a block of niobium $230 \mathrm{~mm} \times 110 \mathrm{~mm}$ and $3 \mathrm{~mm}$ thick provided by AMAC. Holes were punched into the sample. Small tantalum grains of known size were placed into the holes. An electron beam is then used to melt the niobium, covering the tantalum pieces. 


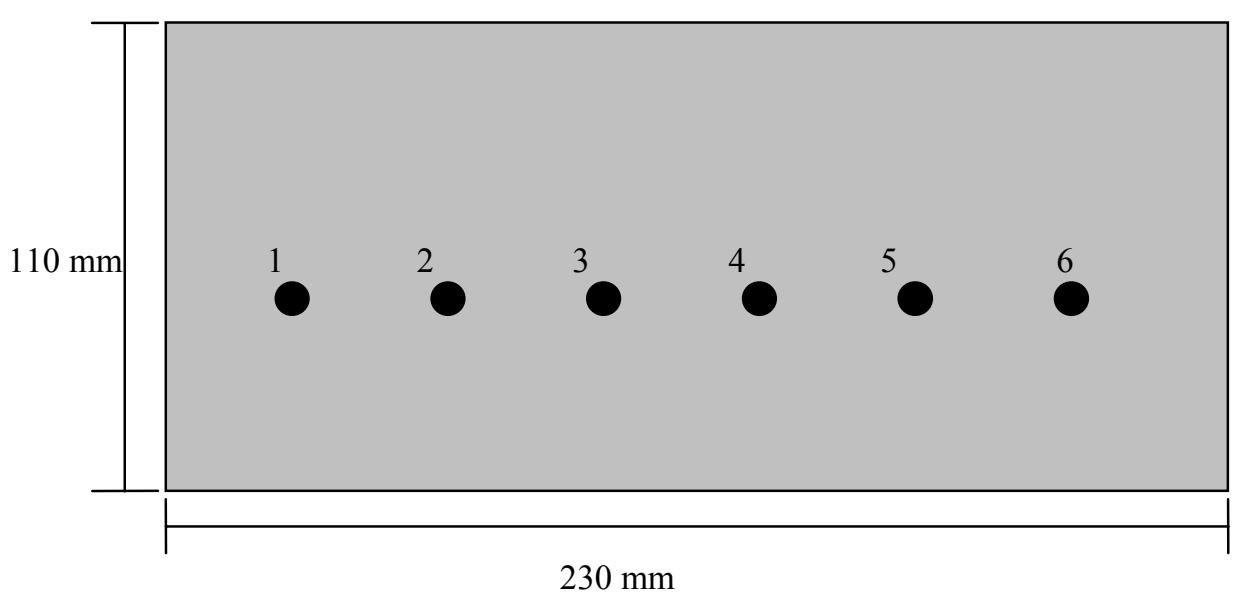

Figure 14 - Niobium block with known Tantalum inclusions Thickness $=3 \mathrm{~mm}$

Inclusion $1=100 \mu \mathrm{m}$ diameter, $100 \mu \mathrm{m}$ deep

Inclusion $2=100 \mu \mathrm{m}$ diameter, $200-300 \mu \mathrm{m}$ deep

Inclusion $3=100 \mu \mathrm{m}$ diameter, $200 \mu \mathrm{m}$ deep

Inclusion $4=100 \mu \mathrm{m} \times 150 \mu \mathrm{m}, 100 \mu \mathrm{m}$ deep

Inclusion $5=100 \mu \mathrm{m}$ diameter, $200 \mu \mathrm{m}$ deep

Inclusion $6=200 \times 150 \mu \mathrm{m}, 100 \mu \mathrm{m}$ deep

We used the SQUID system described earlier to scan this sample in a continuous mode. The induced current for scanning was fairly large in the range from $0.5-1.0$ Amps in order to produce the largest possible signal from the defect and the frequency used was in the range from $8 \mathrm{k} \mathrm{Hz}$ to $48 \mathrm{~K} \mathrm{~Hz}$. The scanning speed used typically was from $0.1 \mathrm{~mm} / \mathrm{s}$ to $1 \mathrm{~mm} / \mathrm{s}$ although the scanning speed can be easily increased to $10 \mathrm{~mm} / \mathrm{s}$.

The results of figure 18 below were taken using an excitation current of 0.55 Ampere through the inducer at frequency of $40 \mathrm{k} \mathrm{Hz}$. The scanning speed for this data is 0.3 $\mathrm{mm} / \mathrm{s}$. The results shown in figure 18 are the best for the system as the environmental noise was the lowest while scanning at that time. Environmental noise from $60 \mathrm{~Hz}$ power and the lab environment is one of the primary targets for improvement of this system, namely by adding a magnetic shield to improve the performance of the SQUID sensor.

Figure 18 shows a scan of the AMAC sample with successful detection of defects $\# 5$ and defect \#4. Defect \#5 at the bottom of the graph shows the largest intensity. It is very clear from this intensity chart that the SQUID system can clearly detect a defect with 100 micron diameter located 200 micron below the surface of the sheet. 


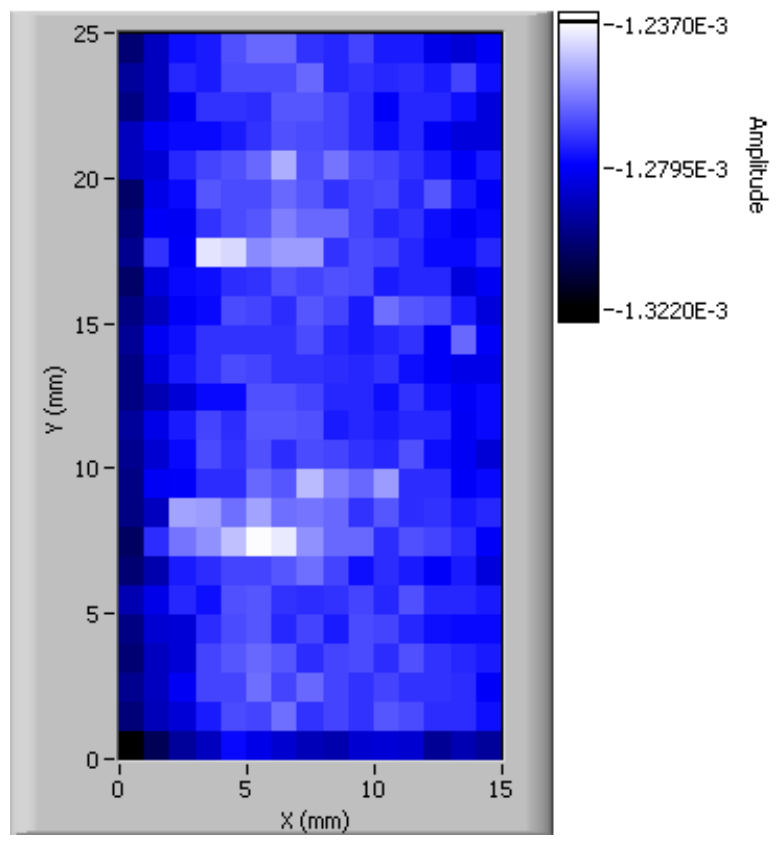

\section{Figure 18: Scan results for defects 5 and 4 of the AMAC Nb sheet.}

Figure 19 shows the same scan data acquired by the system for defects \#5 through defect $\# 1$. In this larger size scan one can also identify all 5 defects on the same scan, although the intensity of the signal varies from a defect to a defect due to the variation in size and depth of the defect. A data filtering software can enhance the signal from the defects and make it easier for the operator of the system to identify these defects.

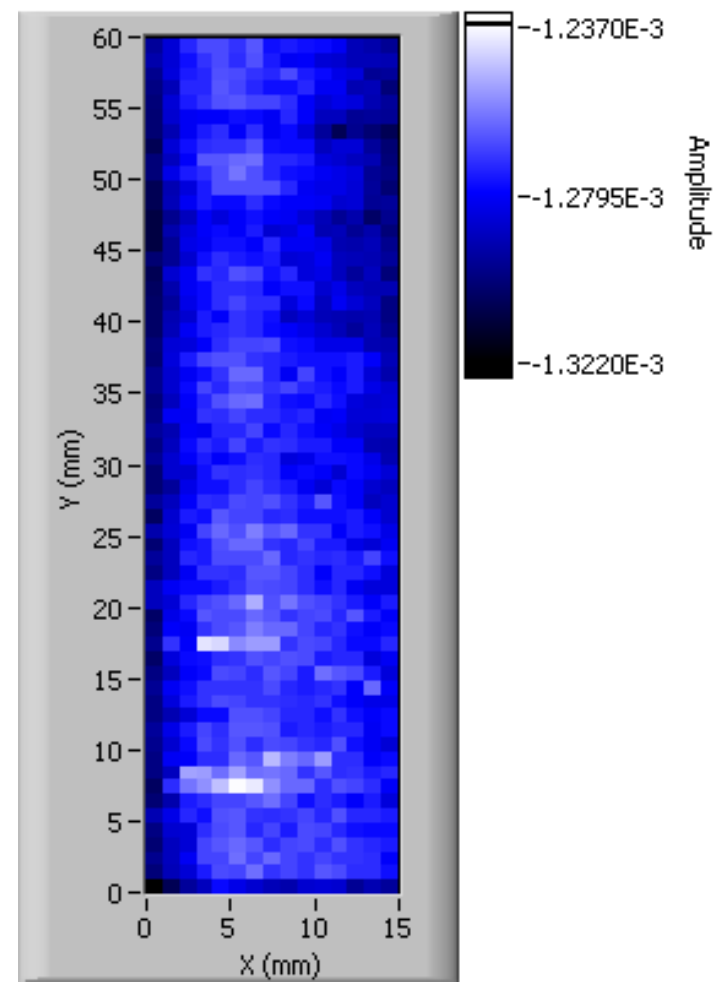


Figure 19: Scan results for defect 5 through defect 1 in the AMAC Nb sheet.

\section{2) Flat Nb sheet made by the DESY group}

The flat sample used in this project was provided by DESY in Figure 20. DESY produced this sample in a similar fashion to the AMAC sample by placing tantalum into holes and melting niobium over the inclusion.

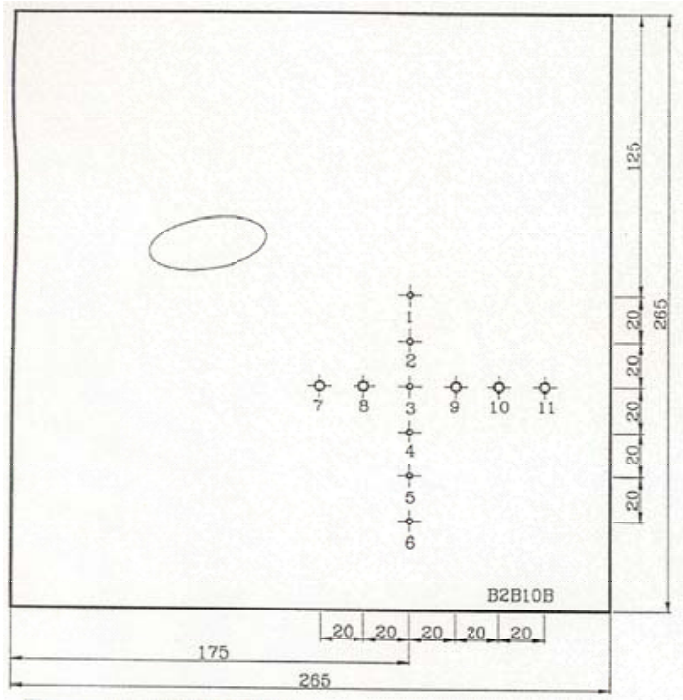

Figure 20. Schematic of DESY Sample

Table 2 - A description of the inclusions within the DESY sample.

\begin{tabular}{|r|r|r|l|l|}
\hline \multicolumn{4}{|l|}{ Description of DESY sample } & \multicolumn{2}{|l|}{$\left.\mathrm{Nm}^{3}\right)$} \\
\hline Hole & Diameter $(\mathrm{mm})$ & Depth $(\mathrm{mm})$ & Volume of Ta Inclusion $\left.\mathrm{mm}^{3}\right)$ \\
\hline 1 & 0.12 & 0.078 & 0.0009 \\
\hline 2 & 0.14 & 0.21 & 0.0032 \\
\hline 3 & 0.145 & 0.298 & 0.0049 \\
\hline 4 & 0.145 & 0.376 & 0.0062 & \\
\hline 5 & 0.15 & 0.474 & 0.0084 & \\
\hline 6 & 0.15 & 0.469 & 0.0083 & \\
\hline 7 & 0.22 & 0.234 & 0.0089 \\
\hline 8 & 0.23 & 0.333 & 0.0127 & \\
\hline 9 & 0.22 & 0.435 & 0.0165 & \\
\hline 10 & 0.22 & 0.495 & 0.0188 & \\
\hline 11 & 0.22 & 0.501 & 0.019 & \\
\hline
\end{tabular}

The sample from the DESY group has defects of comparable size to the $\mathrm{Nb}$ sample made by AMAC. 
The results of figure 21 below was taken using an excitation current of $0.68 \mathrm{~A}$ through the inducer at $40 \mathrm{k} \mathrm{Hz}$. The scanning speed for this data is $0.25 \mathrm{~mm} / \mathrm{s}$. The plate was placed with the defects placed close to pick up coil. The chart shows the scan of defects \#6 through defect \#1.

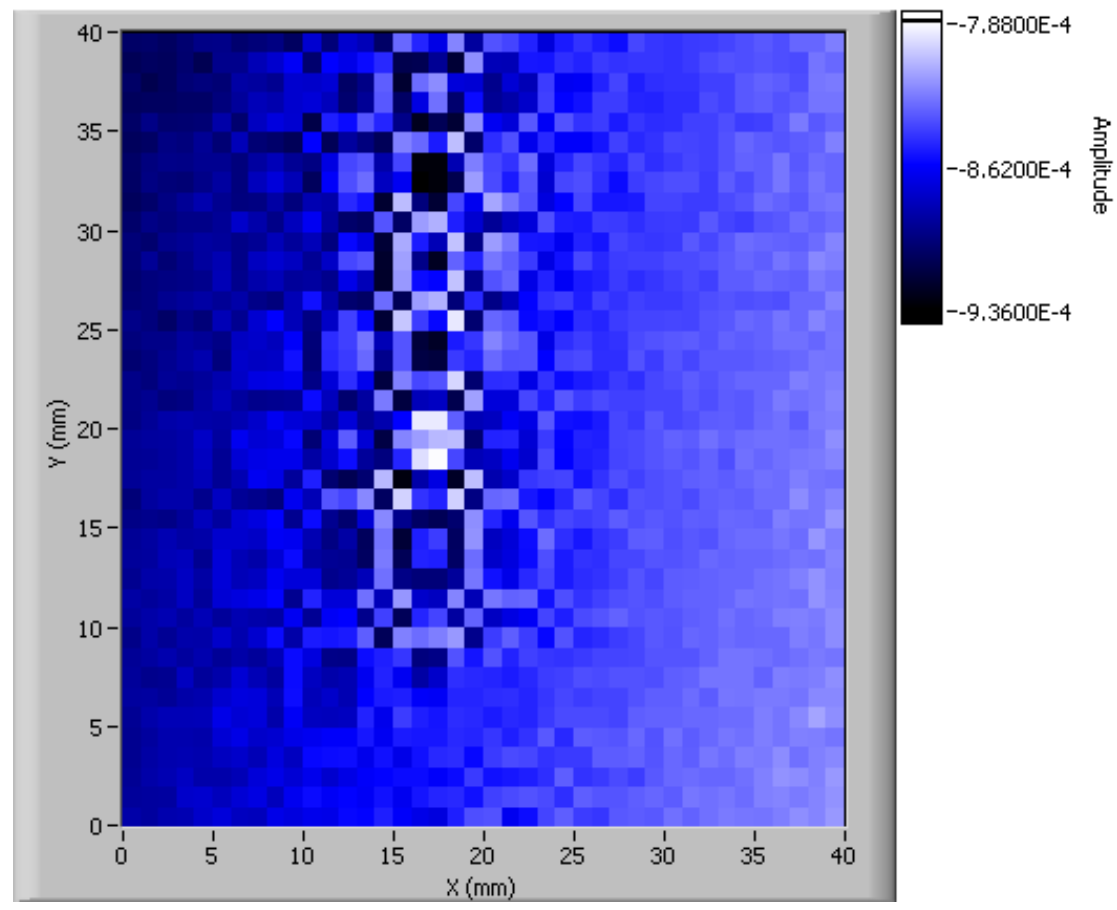

Figure 21. DESY sample with defects close to the pick coil

The results shown in figure 22 below were taken using an excitation current of 0.68 Amperes through the inducer at $12 \mathrm{KHz}$. The scanning speed for this data is $0.25 \mathrm{~mm} / \mathrm{s}$. 


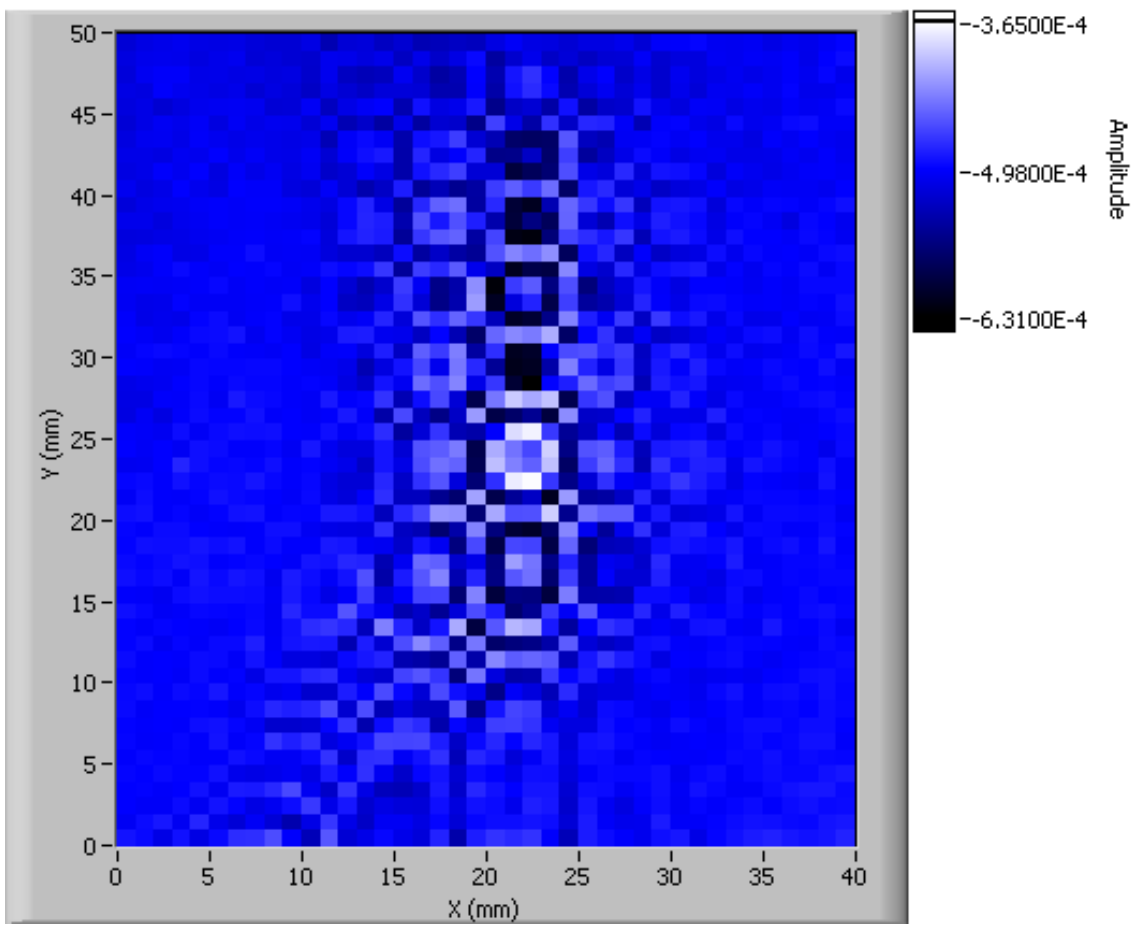

\section{Figure 22: scan results of defects \#6 through \#1 of the DESY sample}

This figure shows that the SQUID system detected the presence of Ta defects \# 6 through \#1 of the DESY sample. Clearly the data is very pixilated in this graph too indicating the need for higher sensitivity. This can be accomplished by increasing the diameter of the pick up coil for the SQUID which might lead to an increase in the flux linked to the Squid and higher sensitivity. The dark and while areas are typical of a signal by the eddy current technique described earlier in the introduction and are strong indicators for the presence of the defects.

Another representative data from our results is shown in figure 23. This scan was performed with the sample flipped i.e. defects are on the bottom side of the sheet and further away from the pick up coil and the current inducer. This resembles the scanning of the inside surface of $\mathrm{Nb}$ half-cell where the pick up coil of the SQUID would be further away from the defects.

The excitation current used is 0.72 Ampere at $12 \mathrm{KHz}$ with a scanning speed of 0.25 $\mathrm{mm} / \mathrm{s}$. The figure shows the signal detected from defect \#1 which is the smallest defect of the DESY sample with a diameter of 120 microns. 


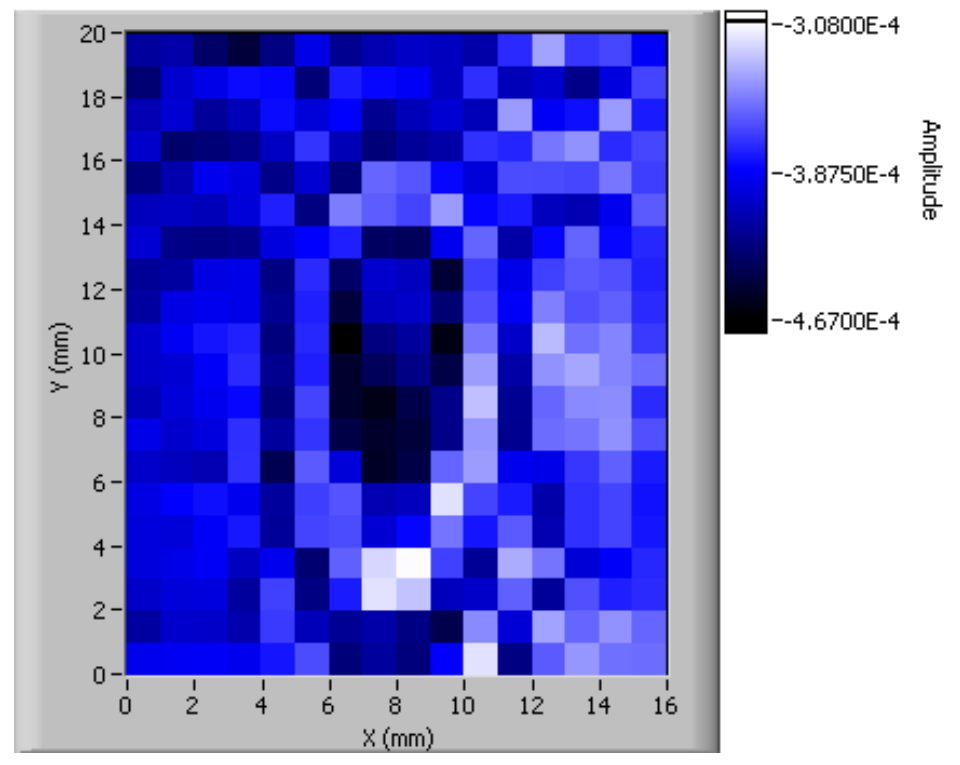

Figure 23: The smallest defect of the DESY sample (Defect \#1) detected at the bottom side of the $\mathrm{Nb}$ sheet

\section{H. CONCLUSION}

The feasibility studies have been successfully completed with very positive results. We have developed a fast scanning SQUID- based system that replaced an existing system that was too slow. In phase I work, we needed to scan in a continuous mode without stopping the scanning table at $1 \mathrm{~mm}$ intervals to take readings. We designed and implemented a SQUID scanning system that is fast and is capable of scanning a $30 \mathrm{~cm} \mathrm{x}$ $30 \mathrm{~cm} \mathrm{Nb}$ sheet in 15 minutes by continuously moving the table at speeds up to $10 \mathrm{~mm} / \mathrm{s}$ while activating the SQUID at $1 \mathrm{~mm}$ interval. The Dewar, SQUID probe and controller was provided by Tristan Technologies (San Diego, CA.) based on the specifications of our team. The SQUID output is connected to a Stanford Research SR530 lock-in amplifier to isolate the signal produced by the eddy current around the defect. We have installed a new scanning table, which uses a screw mechanism to greatly reduce mechanical vibrations and allow us to scan in continuous fashion. We have also developed custom data acquisition Labview software to fully automate the measurement. This custom data acquisition system allows for choosing the scanning speed in the range from $0.1 \mathrm{~mm} / \mathrm{s}$ to $10 \mathrm{~mm}$, update the position of the scanning table and activate a measurement at uniform intervals of $1 \mathrm{~mm}$. Results show that we can scan a large portion of a $30 \mathrm{~cm} \times 30 \mathrm{~cm} \mathrm{Nb}$ sheet in a continuous fashion and detect Ta defect of 100micron size. We have successfully demonstrated that we scan at fast speeds without sacrificing the resolution of detection. Custom data analysis software was also developed to address the lift off effect that is the result of having a slightly uneven gap between the $\mathrm{Nb}$ sheet and the pick up coil of the SQUID.

The emphasis for phase II will be on improving the sensitivity of the SQUID system by 1) refining the squid specifications and increasing the flux linkahe between the sample and the pick up coil. 2) improving the magnetic shielding of the system which will 
consideribly reduce the environmetal noise interfering with the SQUID and true defect signal. 3) designing a sample mounting system to ensure that the gap between the $\mathrm{Nb}$ sheet and tail of the dewar is small and more even. These three hardware design changes will improve the quality of the data collected, increase sensitivity and make is it easier the extract the real signal from the defect enabling us to reach our goal of detecting defects less than 50 micron in diameter. We will also develop a filter and defect- reconignition software system for the analysis of the data. Finally we will deliver a protype scanning system that is faster, more sensitive and has fully integrated hardware and software.

\section{REFERENCES}

1) Singer W, Brinkmann A, Proch D, Singer X; Physica C, 386(2003)379-384

2) Jenks W G, Sadeghi S S H, Wikswo J P; J. Phys. D, 30(1997)293-323

3) Selim R, McFarlane M, Mast J, Wincheski B, Simpson J; Review of Progress in Quantitative Nondestructive Evaluation, eds. Thomas D O, Chimenti D E, Vol. 24B, P. 1638, Plenum Press, New York, NY

4) Ma Y P, Wikswo J P; Review of Progress in Quantitative Nondestructive Evaluation, eds. Thomas D O, Chimenti D E, Vol. 17, Plenum Press, New York, NY

5) Shu Q.-S., Susta J., Cheng G. F., Phipps I. , Selim , R., Mast J. , Kneisel P., Myneni G. and Ben-Zvi I.; IEEE Proceedings of Particle Accelerator Conference, P. 2469-2471, Albuquerque, New Mexico, June 2007 


\section{J. Appendix A: User Manual for SQUID Data Acquisition System}

Select the VI "SQUID_acquisition_March08.vi" which is the final VI for reading and collecting the data.

At the front panel, there are seven inputs and five outputs. Inputs are: Board ID, Seconds to wait, $\mathrm{X}$ distance $(\mathrm{mm}), \mathrm{Y}$ distance $(\mathrm{mm})$, Velocity $(\mathrm{mm} / \mathrm{s})$, Acceleration (RPS/S), Resolution (mm), file path, and Stop button. Outputs are: X position (mm), Y POSITION (mm), Velocity (RPM) and two graphs $\mathrm{X}(0)$ and $\mathrm{X}(90)$. The number of data points in the $\mathrm{X}$ axis is: $\mathrm{X}$ distance $(\mathrm{mm})$ / Resolution $(\mathrm{mm})$ and the number of lanes in $\mathrm{Y}$ axis is: $\mathrm{Y}$ distance $(\mathrm{mm}) /$ Resolution $(\mathrm{mm})$.

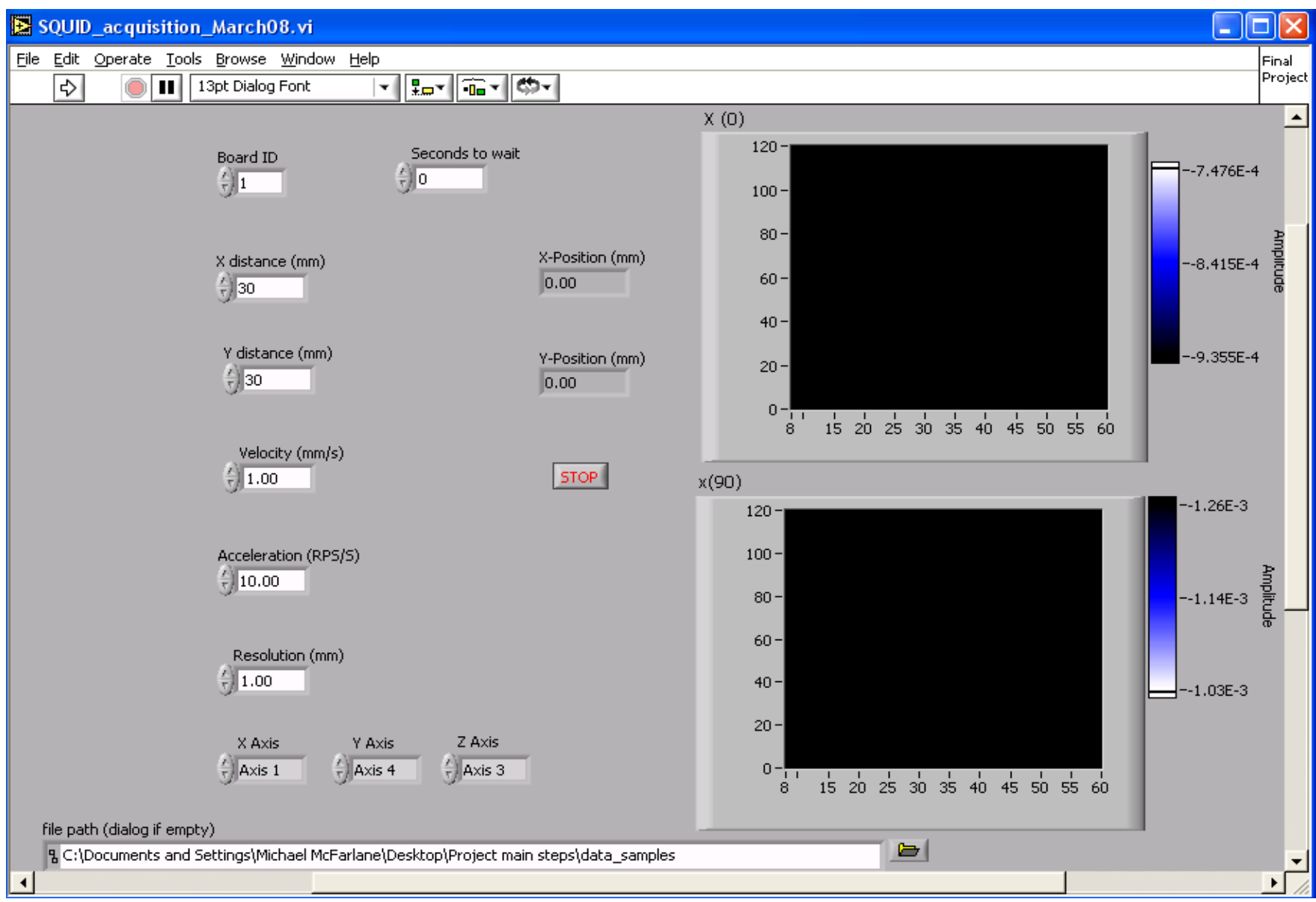

Make sure that the board ID is 1 and Acceleration is 10 RPS/S. The control variable seconds to wait represents the amount of time to wait after the SQUID has been reset; it is set as default to 0s. Afterwards, enter the $\mathbf{X}$ distance (mm), $\mathbf{Y}$ distance (mm), Velocity, and Resolution. Also, specify the file path, located at the bottom of the front panel, where the data to be collected will be saved.

Note: Since the velocity relates to the range of collecting the data (the range specifies the boundaries where the data can be taken), which was set 
to be + or -5 counts $=0.14 \mathrm{~mm}$, it is preferable not to exceed $15 \mathrm{~mm} / \mathrm{s}$ in the velocity for accurate results. Also, the default value of resolution should be set to $\mathbf{1} \mathbf{m m}$, but it is possible to change it to any other desired resolution.

At the bottom of the front panel of the VI, there are three Axis controls, X Axis, Y Axis, and $\mathrm{Z}$ Axis. According to current wiring with the Axis Stepper Motor Drive, they should be set to Axis 1 for X Axis, Axis $\mathbf{4}$ for Y Axis, and Axis $\mathbf{3}$ for Z Axis.

After entering all the inputs, select "Run" located under "Operate" in the toolbar.

An initialization VI will be displayed which allows moving $\mathrm{X}, \mathrm{Y}$ and $\mathrm{Z}$ coordinates; after moving the coordinates to the desired position, this VI allows you to set that position as your starting point for the experiment. The current $\mathrm{X}, \mathrm{Y}$ and $\mathrm{Z}$ positions are displayed in the right indicators; they are also updated every time $\mathrm{X}, \mathrm{Y}$ or $\mathrm{Z}$ are moved.

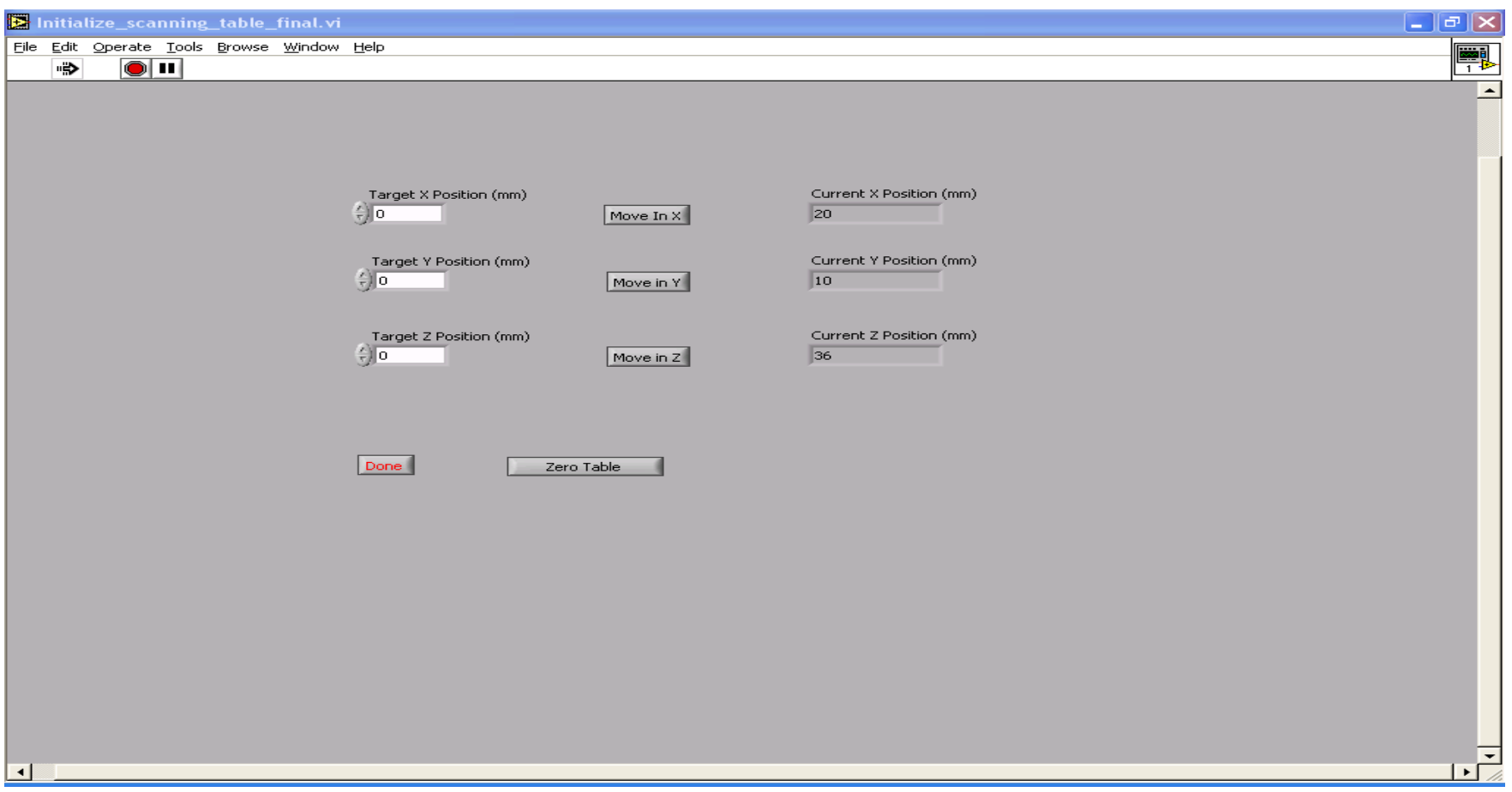

For instance, to move the $\mathrm{X}$ coordinate, enter the $\mathbf{X}$ target position (mm) and press Move In X. Same thing for $Y$ and $Z$.

Note: For the $Z$ axis, the maximum height is $52 \mathrm{~mm}$, so the target $Z$ position should not exceed that value. Also, check the positive and negative directions of $\mathrm{X}$ and $\mathrm{Y}$ axis in the table.

After moving $\mathrm{X}, \mathrm{Y}$ and $\mathrm{Z}$ to the desired position, press "Zero Table" to make that point the origin coordinate of the experiment. Then, press "Done" button to process collecting the data. The data will be collected while the motor is moving in the $\mathrm{X}$ positive direction and it will be displayed on a two dimension graphs at the end of every lane. In addition, the current $\mathrm{X}$ and $\mathrm{Y}$ positions ( $\mathrm{mm}$ ) will be displayed while taking the data. 
In case you want to stop the experiment while it is running, you can hit the "Stop" button, which will stop collecting the data after the current lane and save the data under the specified directory.

The two final displayed graphs are the Lock in readings of $X(0)$ and $X(90)$ and the colors represent the amplitude values.

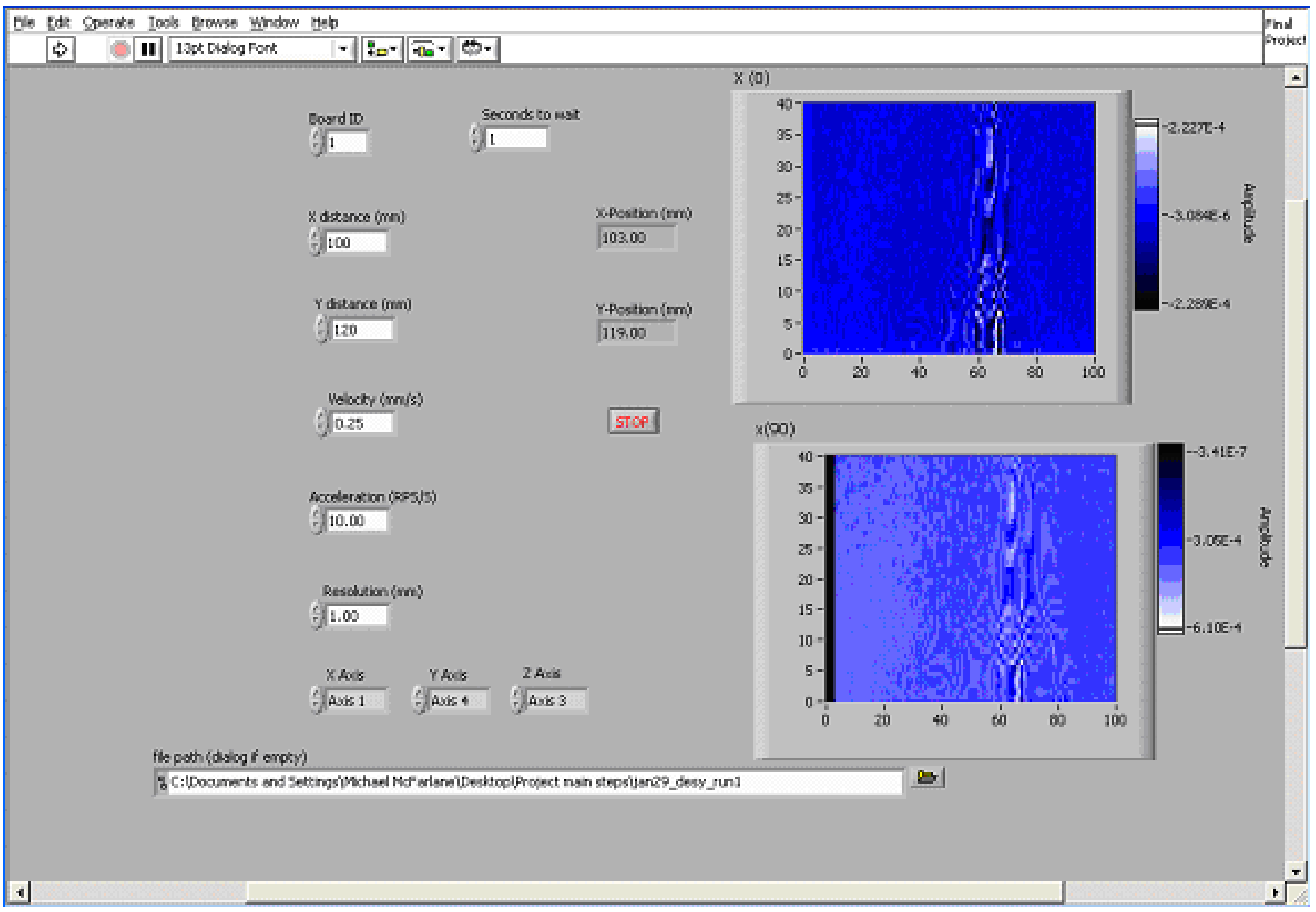

